\title{
Circular RNA cESRP1 sensitises small cell lung cancer cells to chemotherapy by sponging miR-93-5p to inhibit TGF- $\beta$ signalling
}

\author{
Weimei Huang ${ }^{1}$ - Yunchu Yang ${ }^{1}$ Jingfang $\mathrm{Wu}^{1} \cdot{\text { Yuchun } \mathrm{Niu}^{1,2} \cdot \text { Yao Yao }^{3} \cdot \text { Jian Zhang }}^{4} \cdot$ Xiaoxian Huang $^{5}$. \\ Shumei Liang ${ }^{1} \cdot$ Rui Chen $^{1} \cdot$ Size Chen ${ }^{6} \cdot$ Linlang Guo $^{1}$
}

Received: 16 May 2019 / Revised: 30 October 2019 / Accepted: 31 October 2019 / Published online: 14 November 2019

(c) The Author(s) 2019. This article is published with open access

\begin{abstract}
Circular RNAs (circRNAs) are novel RNA molecules that play important roles in chemoresistance in different cancers, including breast and gastric cancers. However, whether circRNAs are involved in the response to chemotherapy in small cell lung cancer (SCLC) remains largely unknown. In this study, we observed that cESRP1 (circular RNA epithelial splicing regulatory protein1) expression was significantly downregulated in the chemoresistant cells compared with the parental chemosensitive cells. cESRP1 enhanced drug sensitivity by repressing miR-93-5p in SCLC. Cytoplasmic cESRP1 could directly bind to miR-93-5p and inhibit the posttranscriptional repression mediated by miR-93-5p, thereby upregulating the expression of the miR-93-5p downstream targets Smad7/p21(CDKN1A) and forming a negative feedback loop to regulate transforming growth factor- $\beta$ (TGF- $\beta$ ) mediated epithelial-mesenchymal transition. Furthermore, cESRP1 overexpression and TGF- $\beta$ pathway inhibition both altered tumour responsiveness to chemotherapy in an acquired chemoresistant patient-derived xenograft model. Importantly, cESRP1 expression was downregulated in SCLC patient tissues and was associated with survival. Our findings reveal, for the first time, that cESRP1 plays crucial a role in SCLC chemosensitivity by sponging miR-93-5p to inhibit the TGF- $\beta$ pathway, suggesting that cESRP1 may serve as a valuable prognostic biomarker and a potential therapeutic target in SCLC patients.
\end{abstract}

These authors contributed equally: Weimei Huang, Yunchu Yang, Jingfang $\mathrm{Wu}$, Yuchun Niu, Yao Yao

Edited by M. Piacentini

Supplementary information The online version of this article (https:// doi.org/10.1038/s41418-019-0455-x) contains supplementary material, which is available to authorized users.

Size Chen

chensize@gdpu.edu.cn

$\triangle$ Linlang Guo

linlangg@yahoo.com

1 Department of Pathology, Zhujiang Hospital, Southern Medical University, Guangzhou, China

2 Department of Oncology, The First Affiliated Hospital of Hebei North University, Zhangjiakou, China

3 Department of Pathology, Peking University Third Hospital, Beijing, China

4 Department of Oncology, Zhujiang Hospital, Southern Medical University, Guangzhou, China

5 Clinical Laboratory, Gushang Hospital of Guangxi Zhuang Autonomous Region, Nanning, China

6 Department of Oncology, The First Affiliated Hospital of Guangdong Pharmaceutical University, Guangzhou, China

\section{Introduction}

Lung cancer remains one of the most common causes of cancer-related mortality worldwide, with small cell lung cancer (SCLC) accounting for $\sim 15 \%$ of all lung cancer cases $[1,2]$. The high death rate of SCLC patients is directly related to the fact that most patients eventually develop resistance to platinum-based chemotherapy and ultimately die from their disease [3]. Thus, there is a drastic need to identify new therapeutic targets or treatments that restore chemosensitivity and improve tumour control in relapsed chemoresistant SCLC patients.

Circular RNAs (circRNAs) are naturally occurring members of the noncoding transcriptome and have a covalently closed loop structure [4]. The functional roles of circRNAs have been well characterised to date, such as in mesenchymal stem cell identity maintenance [5], differentiation [6], development [7], and oncogenesis [8, 9]. CircRNAs can influence various cellular processes including proliferation, cell cycle progression, and cell apoptosis $[10,11]$. More meaningfully, they can act as oncogenes or tumour suppressors through diverse mechanisms to regulate tumour progression $[9,12]$ and tumour resistance to 
chemotherapy $[13,14]$. However, the circRNAs involved in SCLC chemoresistance remain largely unknown.

Transforming growth factor- $\beta$ (TGF- $\beta$ ) is one of the most prominent molecules involved in cancer progression, including SCLC [15, 16]. During cell development and carcinogenesis, TGF- $\beta$ ligand activation results in the phosphorylation of TGF- $\beta$ receptor type I (T $\beta$ RI), Smad2, and Smad3 (Smad2/3) [17]. Subsequently, phosphorylated $\mathrm{Smad} 2 / 3$ form complexes with Smad4 and translocate into the nucleus, binding to specific DNA sequence motifs and regulating the transcription of target genes, including Smad7 [18, 19]. Smad7 can act as negative feedback regulator of the TGF- $\beta$-mediated signalling by inhibiting T $\beta$ RIregulated Smad2/3 phosphorylation or impeding the binding of Smads complexes to DNA sequences $[20,21]$. In addition to regulating Smad7, the p-Smad2/3-Smad4 complex can also interact with additional transcriptional regulators in the nucleus to transactivate downstream target genes of TGF- $\beta$, such as p21(CDKN1A) [22-24]. Upon TGF- $\beta$ signalling activation, a series of processes occur that can lead to cancer development, and TGF- $\beta$ signalling can increase drug resistance in tumour cells by mediating epithelial-to-mesenchymal transition (EMT) [25, 26]. Blocking the TGF- $\beta$ signalling pathway can reverse chemoresistance in tumour cells [27]. In several cancers, such as prostate cancer, glioblastoma, and lung cancer, the TGF$\beta$ receptor I inhibitor galunisertib can sensitise cancer cells to anticancer drugs by suppressing TGF- $\beta$-mediated EMT [28-30]. It has been reported that $\mathrm{p} 21(\mathrm{CDKN} 1 \mathrm{~A})$ is upregulated by TGF- $\beta$-mediated pathways and can repress TGF$\beta$-induced features of EMT by interfering with TGFBR2 expression or reversing twist-mediated E-cadherin promoter repression [31, 32]. However, whether p21(CDKN1A) plays a role in chemoresistance through TGF- $\beta$-mediated EMT remains unknown. Recently, noncoding RNAs, including miRNA and circRNA, have been shown to promote chemoresistance via the TGF- $\beta$-induced EMT pathway [33-36]. However, to date, no studies have reported whether circRNAs can affect SCLC chemoresistance by repressing TGF- $\beta$-induced EMT.

In this study, we screened for circRNAs exhibiting differentially expression between the parental SCLC cell line H69/H69AR and identified a novel circRNA derived from the ESRP1 (RBM35A) gene locus (cESRP1). Low expression of cESRP1 in patients with SCLC was positively associated with poor survival. Further investigation showed a critical role for cESRP1 in inhibiting TGF- $\beta / \mathrm{Smad}$ signalling-induced EMT. We revealed, for the first time, a novel interaction between cESRP1 and a protein complex known to be involved in the negative feedback regulation of TGF- $\beta /$ Smad signalling activation, establishing cESRP1 as an emerging circRNA that is functionally important in SCLC chemosensitivity.

\section{Materials and methods}

\section{Tumour specimens and primary cell cultures}

Patient-derived paraffin-embedded tumour sections were collected from the First Affiliated Hospital of Hebei North University and the Minzu Hospital of Guangxi Zhuang Autonomous Region for use in this study, which was approved by the appropriate ethics committee. Informed consent was obtained from all patients before specimen collection. RNA was extracted from the paraffin-embedded tissue samples to assess the RNA levels of target molecules using an FFPE RNA kit (GBCBIO, Guangzhou, China), and the primers used are provided in the Supplemental Information. The three cell lines (NCI-H69, NCI-H69AR, and NCI-H446) used in this study were purchased from the American Type Culture Collection (ATCC, USA) and cultured in RPMI medium (HyClone; Thermo Scientific, CA, USA) supplemented with $10 \%$ fetal bovine serum (FBS; Gibco, New York, USA) in a humidified atmosphere containing $5 \% \mathrm{CO}_{2}$ at $37^{\circ} \mathrm{C}$. Cell line identity was commercially authenticated via short tandem repeat profiling, which was performed by the Cellcook Biology Company of China. The drug-resistant subline H446DDP was established in our laboratory by culturing H446 cells in gradually increasing concentrations of cisplatin (up to $0.5 \mu \mathrm{g} / \mathrm{ml}$ ) for 12 months and was maintained in complete medium containing cisplatin. The primary cell line PDC1-S was produced from fresh xenograft tumour tissue from patient-derived xenograft (PDX)1-naive mice, and the cell line PDC1-R was produced from PDX1chemoresistant mice; Both cell lines were maintained in Dulbecco's modified Eagle's medium (DMEM)/F-12 (Gibco, New York, USA) supplemented with 10\% FBS (Gibco, New York, USA). The extraction steps used to process primary cells and the reagents used are specifically listed in the Solid tissue disaggregation section. The cell lines used in this study were not contaminated with mycoplasma.

\section{circRNA expression profiles}

Experimental technology was provided by the Shanghai Kangcheng Biological Company (China). Briefly, H69 and H69AR cells were used for circRNA microarray assays. Total RNA was extracted from cell lysates and evaluated for quality by agarose gel electrophoresis. Two micrograms of total RNA were treated with RNase R. After sample labelling, hybridisation, and washing, the samples were analysed using circRNA chips (Arraystar Human circRNAs chip; Arraystar, Rockville, MD, USA). Exogenous RNAs developed by the External RNA Controls Consortium (Applied Biosystems, USA) were used as controls. 
Cell counting kit-8 assay and the determination of $\mathbf{5 0 \%}$ inhibitory concentration (IC50) values

Cells in complete growth medium were inoculated into a 96-well tissue culture plate at a density of 3000-12,000 cells per well. After $24 \mathrm{~h}$ of culturing, growth medium containing chemotherapeutic drugs, including cisplatin (cisplatin injection; Shandong, China), etoposide (Vepesid; Bristol-Meyers Squibb, Australia), and doxorubicin (Hisun Pfizer; Hangzhou, China) was added to the wells. Wells containing drug-free growth medium were used as controls. Then, the plate was incubated for $24 \mathrm{~h}$ before assessing cell viability. Luminescence analysis was performed according to the instructions of the CCK8 manufacturer (Dojindo, Japan), and the 50\% inhibitory concentration (IC50) values of the drugs were calculated using Graphpad.

\section{RNA isolation, treatment with RNase $R$, and quantitative real-time PCR (qRT-PCR)}

Total RNA was extracted from cells and tumour samples using RNAiso Plus* (Takara, Japan) according to the manufacturer's instructions. Cytoplasmic and nuclear RNA was isolated using a Nuclear/Cytoplasmic Isolation Kit (BioVision, San Francisco, USA) according to the manufacturer's instructions. For RNase R treatment, $1500 \mathrm{ng}$ of total RNA was incubated for $30 \mathrm{~min}$ at $37^{\circ} \mathrm{C}$ with or

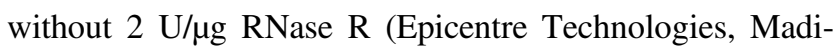
son, WI, USA). cDNA was synthesised using a Fast Quant RT Kit (TIANGEN BIOTECH, Beijing, China) according to the manufacturer's instructions. Then, quantitative realtime PCR (qRT-PCR) was performed using $2 \times$ Talent qPCR PreMix (TIANGEN BIOTECH, Beijing, China) according to the manufacturer's guidelines with a Bio-Rad CFX Connect instrument (Bio-Rad, USA). The relative RNA expression levels were analysed using the $2^{-\Delta \Delta \mathrm{Ct}}$ method, with $\beta$-actin used as an internal reference. The primers and RNA sequences used for qRT-PCR are shown in the Supplemental Information.

\section{Fluorescence in situ hybridisation (FISH)}

FITC-labelled miR-93-5p and Cy3-labelled cESRP1 probes were designed and commercially synthesised by GenePharma (Shanghai, China). The probe sequences are provided in the Supplemental Information. A fluorescence in situ hybridisation (FISH) kit (RiboBio, Guangzhou, China) was used to detect probe signals according to the manufacturer's instructions after culturing cells for $24 \mathrm{~h}$. To determine the cESRP1 status of PDX tumours, 4- $\mu$ m-thick sections were cut from paraffin-embedded blocks and then processed, hybridised, and analysed. Cell nuclei were stained with 4',6-diamidino-2-phenylindole (DAPI; Life Technologies,
Carlsbad, CA, USA), and images were acquired using an LMS 880 confocal microscope (Carl Zeiss, Germany).

\section{Western blotting (WB)}

Cell lysates were prepared using RIPA buffer (CWBIO, Beijing, China) supplemented with a phosphatase inhibitor cocktail (CWBIO, Beijing, China) and a protease inhibitor cocktail (CWBIO, Beijing, China). Protein concentrations were determined using a Bicinchoninic acid (BCA) Protein Assay Kit (Beyotime Biotechnology, Shanghai, China). Lysates were boiled in SDS-PAGE loading buffer (Beyotime Biotechnology, Shanghai, China) for $10 \mathrm{~min}$ at $95^{\circ} \mathrm{C}$, after which $30-50 \mu \mathrm{g}$ of each protein sample was separated by SDS-PAGE and then electrotransferred to PVDF membranes (Millipore, IPVH00010; Billerica, MA, USA). The membranes were then blocked with $5 \%$ bovine serum albumin (BSA; MRC BIOTECH, Beijing, China). Finally, the blots were incubated with the appropriate primary antibody (Supplemental Information) overnight at $4{ }^{\circ} \mathrm{C}$ and followed by the corresponding HRP-conjugated secondary IgG antibody for $1 \mathrm{~h}$ at room temperature. Chemiluminescence WB reagents (Millipore Corporation, Billerica, MA, USA) were used to detect immunocomplexes. $\beta$-actin served as the loading control in this study.

\section{Pulldown assay using a biotinylated miRNA}

The capture of miR-93-5p-bound competing endogenous RNAs (ceRNAs) in a pulldown assay using biotinylated miR-93-5p was performed as previously described [37]. Briefly, $5-10 \times 10^{6}$ cells were transfected with $40 \mu \mathrm{g}$ of a biotinylated miR-93-5p mimic (RiboBio, Guangzhou, China) using Lipofectamine ${ }^{\mathrm{TM}} 3000$ Transfection Reagent (Invitrogen, CA, USA) according to the manufacturer's instructions. A biotinylated negative mimic served as a control. The cells were harvested for the pulldown experiment $24 \mathrm{~h}$ after transfection. Biotin-coupled RNA complexes were pulled down by incubating the cell lysates with streptavidin-coated magnetic beads (Dynabeads ${ }^{\circledR} \mathrm{M}-280$, Life Technologies, USA \& Canada) on a rotator at $4{ }^{\circ} \mathrm{C}$ overnight. The next day, the M-280 Dynabead-miRNA mixture was washed with a lysis buffer five times, after which the bound RNA was treated with TRIzol for RNA extraction and purified using a phenol:chloroform:isoamyl alcohol mixture (Millipore Corporation, Billerica, MA, USA). Finally, purified RNA was analysed by qRT-PCR.

\section{Histological evaluation and immunohistochemical staining}

Tissue samples embedded in paraffin were cut into serial $4-\mu m$-thick sections, and then the sections were baked at 
$65^{\circ} \mathrm{C}$ for $2 \mathrm{~h}$, deparaffinized in three changes of xylene and rehydrated through 5-min incubations in 100, 95, 85, and $75 \%$ ethanol solutions. Then, the sections were rinsed in phosphate-buffered saline (PBS) for $5 \mathrm{~min}$. For histological examination, sections were stained with haematoxylin and eosin (LEAGENE, Beijing, China) for 3-10 min. Immunohistochemical staining was performed using the Streptavidin-Peroxidase Kit (ZSBIO, Beijing, China) according to the manufacturer's instructions. Briefly, antigen retrieval was performed using Sodium Citrate + EDTA buffer at $95-100{ }^{\circ} \mathrm{C}$ for $8 \mathrm{~min}$, after which the sections were cooled to room temperature. After rinsing in PBS three times for $5 \mathrm{~min}$ each time, endogenous peroxidase activity was blocked using $1 \% \mathrm{H}_{2} \mathrm{O}_{2}$ for $15 \mathrm{~min}$, followed by blocking of the nonspecific binding sites with goat serum for $30 \mathrm{~min}$ at room temperature. Then, the sections were incubated with a specific primary antibody overnight at $4{ }^{\circ} \mathrm{C}$. The next day, the sections were rinsed in PBS three times for $5 \mathrm{~min}$ each time, which was followed by an incubation with a biotinylated secondary antibody for $30 \mathrm{~min}$ at room temperature. Each section was rinsed in PBS three times and incubated with streptavidin-conjugated HRP for $30 \mathrm{~min}$ at room temperature. HRP activity was detected using diaminobenzidine tetrahydrochloride (DAB), and nuclei were distinguished by haematoxylin staining. Dehydration was accomplished by incubating the sections in $75,85,95$, and $100 \%$ ethanol solutions for $5 \mathrm{~min}$ for each solution. In the last step, the sections were cleared in xylene and sealed with neutral gum. Images were acquired using a Leica DM2500 microscope (Leica, Germany). A detailed list of the antibodies used is included in the Supplemental Information.

\section{Immunofluorescence assays}

Cells were seeded on slides for $24 \mathrm{~h}$ and then treated as indicated in the figures. The cells were fixed in $4 \%$ paraformaldehyde for $30 \mathrm{~min}$ and rinsed in PBS three times for $5 \mathrm{~min}$ each time. Subsequently, the slides were incubated in $0.5 \%$ Triton X-100 for $15 \mathrm{~min}$ at room temperature for permeabilization, and nonspecific protein binding was blocked by incubating the cells with goat serum for $30 \mathrm{~min}$ at room temperature. Then, the cells were incubated with a primary antibody overnight at $4{ }^{\circ} \mathrm{C}$. After rinsing in PBS three times for $5 \mathrm{~min}$ each time, the cells were incubated with a dye-conjugated secondary antibody for $1 \mathrm{~h}$ at room temperature. Then, the cells were washed three times in PBS and incubated with DAPI for $10 \mathrm{~min}$ at $37^{\circ} \mathrm{C}$ before being washed in PBS three times for $5 \mathrm{~min}$ each time. An anti-fluorescence quencher (Solarbio, Beijing, China) was used in the last step, and images were acquired using a confocal microscope. A detailed list of the antibodies used is provided in the Supplemental Information.

\section{RNA immunoprecipitation (RIP) assay}

RIP assays were performed using a Magna RIPTM RNABinding Protein Immunoprecipitation Kit (Millipore Corporation, Billerica, MA, USA) as previously described [38]. Briefly, SCLC cells were cultured in $75-\mathrm{cm}^{2}$ cell culture flasks for $24 \mathrm{~h}$ and then harvested and lysed using RIP lysis buffer. Five micrograms of argonaute 2 (Ago2) antibody or normal rabbit $\operatorname{lgG}$ (Negative control; Millipore, MA, USA) was pre-incubated with Magnetic beads to form a magnetic bead-antibody complex; then, cell lysates were incubated with the magnetic bead-antibody complex overnight at $4{ }^{\circ} \mathrm{C}$. Subsequently, the RNA in the immunoprecipitates was purified according to the kit protocol from the manufacturer. The extracted RNA was then analysed by qRT-PCR.

\section{Transient transfection and luciferase reporter assays}

An miRNA mimic and an miRNA inhibitor were purchased from RiboBio. siRNAs specific for cESRP1 but not for mESRP1 as well as a full-length cESRP1 clone in the vector pcDNA3.1 were purchased from GenePharma. The cESRP1 overexpression or cESRP1-siRNA constructs were packaged into adenovirues by GenePharma. Plasmids and oligonucleotides were transfected using Lipofectamine ${ }^{\mathrm{TM}}$ 3000 Transfection Reagent (Invitrogen, CA, USA). For the luciferase reporter assays, the pREL-RB-TGF-beta plasmid was commercially synthesised by RiboBio and was used to verify TGF- $\beta$ transcriptional activity. GV272 luciferase reporter constructs containing a wild-type (WT) or mutant cESRP1 sequences were obtained from the Shanghai GeneChem Corporation of China and used to evaluate the ceRNA activity of cESRP1, where the GV045 plasmid used as an internal control. SCLC cells were transiently transfected with the indicated constructs and a Renilla luciferase plasmid as an internal control. For the TGF- $\beta$ activity assay, $24 \mathrm{~h}$ after transfection, cells were serum starved for $8 \mathrm{~h}$ before stimulation with $10 \mathrm{ng} / \mathrm{ml}$ of human recombinant TGF- $\beta 1$ (Selleckchem, Houston, TX, USA), with luciferase activities were quantified $14 \mathrm{~h}$ later using a dual-luciferase assay (Promega, Germany). For the ceRNA activity assay, $24 \mathrm{~h}$ after transfecting cESRP1 clone plasmids, miR-93-5p mimics were reintroduced and the cells were cultured for an additional $12 \mathrm{~h}$, followed luciferase activity measurement. The luciferase values shown in the figures are representative of independent transfection experiments that were performed independently at least three times. siRNA sequences are provided in the Supplemental Information. 


\section{Establishment of cell lines with stable cESRP1 knockdown or overexpression}

Construction of the pLVX-puro plasmids and packaging of the cESRP1 overexpression and cESRP1 knockdown lentiviruses were performed at GenePharma. SCLC cells were seeded in 24-well plates. When the cell fusion rate reached $40-60 \%$, an MOI (multiplicity of infection) of 50 of lentivirus was added and cells were cultured in complete medium containing $1 \mu \mathrm{g} / \mathrm{ml}$ polybrene for 12-24 h. The transfected cells were maintained in complete medium containing $2 \mu \mathrm{g} / \mathrm{ml}$ puromycin (Solarbio, Beijing, China).

\section{miRNA expression profiles}

The microarray assay was performed by LC Sciences (Texas, USA). Briefly, 2-5 $\mu \mathrm{g}$ of total RNA was extracted and an oligonucleotide tag was then ligated to the poly(A) tails for later fluorescent dye staining. Subsequently, hybridisation was performed on a $\mu$ ParafloTM microfluidic chip using a micro-circulation pump (Atactic Technologies) [39] according to the instructions of manufacturer. PGR (photogenerated reagent) chemistry was used to detect the probes. After hybridisation, the detection was performed using fluorescence labelling with tag-specific $\mathrm{Cy} 3$ and $\mathrm{Cy} 5$ dyes, and hybridisation images were collected using a laser scanner (GenePix 4000B, Molecular Device). The ratio of the two signals ( $\log _{2}$ transformed, balanced) and $p$ values of the $t$-test were calculated, with significantly different signals identified as those with $p$ values of less than 0.01 .

\section{Flow cytometry analysis}

To analyse cell apoptosis, after drug treatment, cells were harvested by digestion with trypsin (Gibco, New York, USA) and washed three times with PBS containing $2 \%$ FBS and $2 \%$ BSA. Then, the cells were incubated with Annexin-V-APC (eBioscience, Thermo Fisher Scientific, CA, USA) for $30 \mathrm{~min}$ at $4{ }^{\circ} \mathrm{C}$. The cells were rinsed three times in PBS, followed by a 5-min incubation in Fixable Viability Dye eFluor ${ }^{\mathrm{TM}} 780$ (eBioscience, Thermo Fisher Scientific, CA, USA). For cell cycle analysis, the cells were fixed in $70 \%$ ice-cold ethanol for $2 \mathrm{~h}$ and stained with propidium iodide (PI, Keygen, Jiangsu, China) in the presence of RNase A (Qiagen, Germany). Fluorescence intensity was measured using a FAC Scan (BD Biosciences, USA). Apoptotic cells were defined as cells with Annexin V-positive staining. The percentages of cells in the G0-G1, S, and G2-M cell cycle phases were counted and compared.

\section{Animal experiments}

\section{SCLC cell line-derived tumour xenograft model}

Research involving animals was performed in compliance with the policies of the animal ethics committee of the Southern Medical University of China. Female BALB/c nude mice aged 3-4 weeks were used for tumour implantation experiments. SCLC cells were resuspended in $100 \mu \mathrm{l}$ of PBS and injected subcutaneously into the flanks of the nude mice. At 7-10 days after implantation, when the tumours became palpable with a diameter of $\sim 5 \mathrm{~mm}$, drugs were intraperitoneally (i.p.) or intravenously (i.v.) injected. For the in vivo administration of the chemotherapeutic drugs cisplatin and etoposide, cohorts of tumour-bearing animals were treated weekly with cycles of cisplatin (cisplatin injection; Shandong, China; $2.5 \mathrm{mg} / \mathrm{kg}$, i.p. injection on day 1) and etoposide (Vepesid; Bristol-Meyers Squibb, Australia; $4 \mathrm{mg} / \mathrm{kg}$, i.p. injection on days $1-3$ ). For the miRNA antagomir treatment, $50 \mu \mathrm{l}$ of the miR-93-5p or control antagomir (diluted in PBS to $2 \mathrm{mg} / \mathrm{ml}$, administered on days 1-3, weekly) was i.v. administered, and LY2157299 (2 mg/kg, days 1-3, weekly) was i.p. injected alone or in combination with the chemotherapeutic drugs (cisplatin, $1.5 \mathrm{mg} / \mathrm{kg}$, day 1, i.p.; and etoposide, $3 \mathrm{mg} / \mathrm{kg}$, days $1-3$, i.p.). Tumour size and mouse body weight were measured with electronic calipers and an electronic scale, respectively, every 3-4 days. Tumour volume was calculated using on the following formula: $\left(\right.$ length $\left.\times w_{i d t h}^{2}\right) / 2$.

\section{Establishment of the PDX model}

PDX models using samples derived from SCLC patients have been described elsewhere [40-42]. To collect fresh tissues, patients treated for SCLC at the Guangdong Provincial People's Hospital and the Zhujiang Hospital of Southern Medical University provided informed consent to the Institutional Review Board. All mouse studies were conducted according to Institutional Animal Care and Use Committee (IACUC)-approved animal protocols in accordance with the Southern Medical University's institutional guidelines. The diagnosis of SCLC was confirmed by a pathologist. To establish a PDX model (P0), SCLC tumour samples (primary surgical tumour specimens or metastatic lymph node resection specimens) were cut into 3-4 mm pieces and subcutaneously transplanted within $4 \mathrm{~h}$ after surgical removal into 3-5 severely immunodeficient B$\mathrm{NDG}^{\circledast}$ mice (BIOCYTOGEN, Beijing, China). The mice were observed daily to assess tumour growth and animal health status. Palpable tumours were measured weekly with electronic callipers until the tumour size exceeded $1000-1500 \mathrm{~mm}^{3}$. At this time, the animals were euthanized, and the tumours were removed. Subsequently, scalpel- 
A

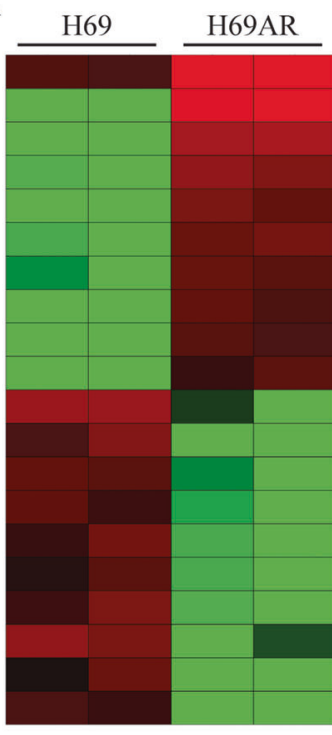

hsa circ 0038090

hsa_circ_0038138 hsa circ 0002346 hsa_circ_0049657 hsa circ 0039929 hsa_circ_0000042 hsa circ 0075048 hsa_circ_0006633 hsa circ 0000230 hsa circ 0005579 hsa_circ_0061276 hsa circ 0000792 hsa_circ_0013912 hsa circ 0002274 hsa circ 0021553 hsa circ 0029614 hsa_circ 0032708 hsa_circ_0084927 hsa circ 0035445 hsa_circ_0086088
B

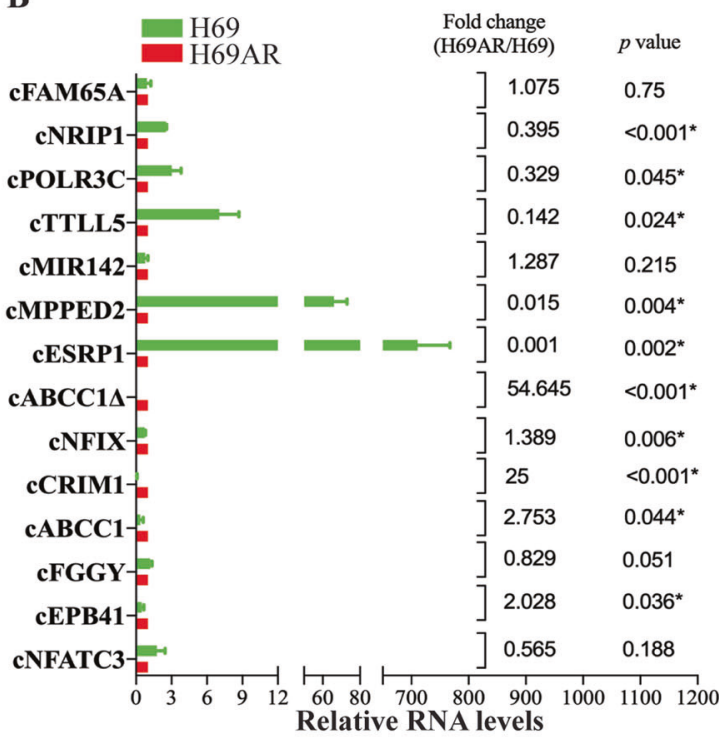

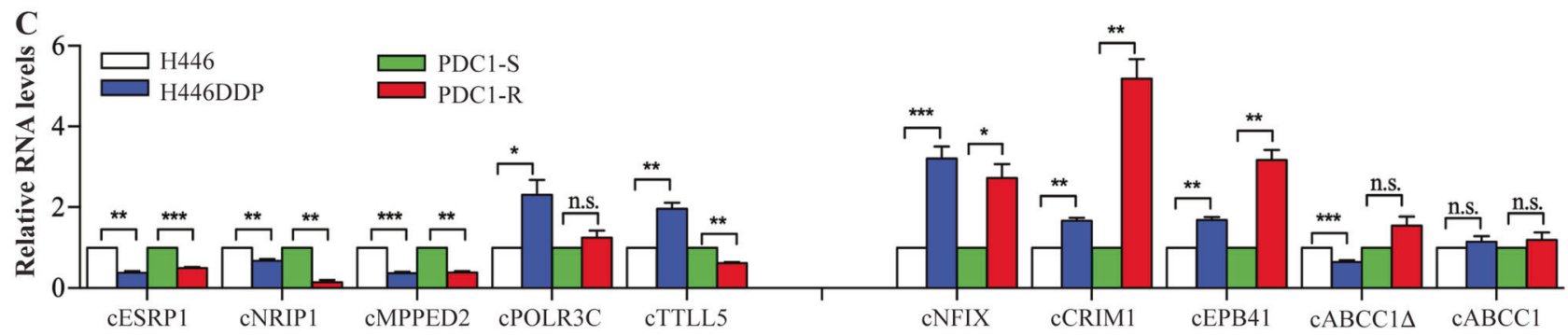

D

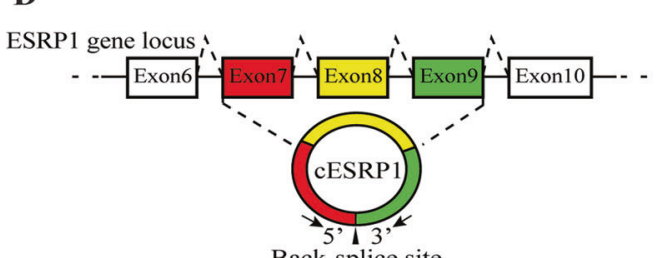

Back-splice site
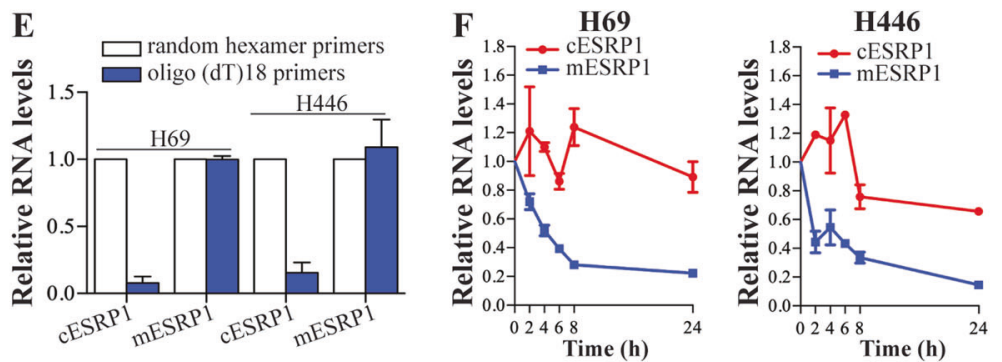

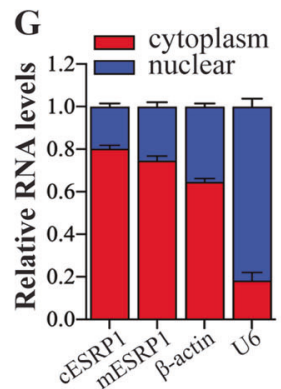

H

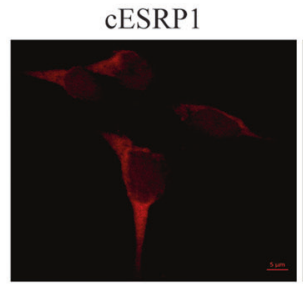

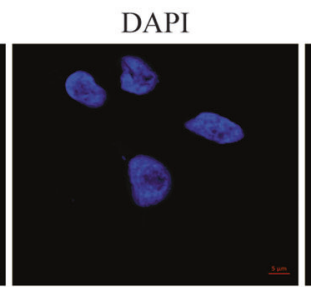
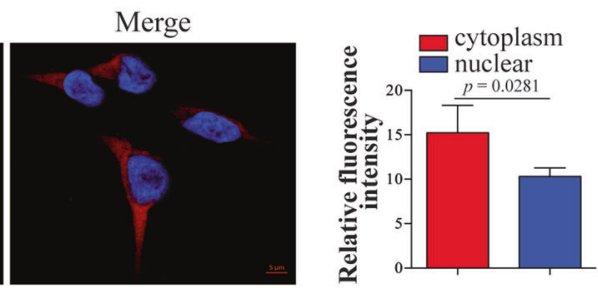

dissected xenograft fragments were either immediately implanted into new $\mathrm{NDG}^{\circledR}$ mice for passaging, digested into single-cell suspensions and cryopreserved in liquid nitrogen for later passaging, fixed in $4 \%$ paraformaldehyde (YongJin Biotech, Guangzhou, China) for pathological analysis, or flash-frozen in liquid nitrogen for molecular analysis.

\section{Solid tissue disaggregation}

Solid tissue samples collected from mouse xenografts were mechanically and enzymatically disaggregated into singlecell suspensions as previously described [43, 44]. Briefly, the solid tissue samples were minced with scissors into 
Fig. 1 Deregulated circRNAs in chemoresistant SCLC and cESRP1 characterisation. a The heatmap shows the top ten circRNAs with the most increased and decreased expression in H69AR cells compared with $\mathrm{H} 69$ cells, as determined using a circRNA Arraystar Chip. b We validated the differential expression of 14 circRNAs in H69AR cells and H69 cells using qRT-PCR. An independent-sample $t$-test was used; qRT-PCR, quantitative reverse transcription PCR. Data are mean $\pm S D$, $n=3$. $\mathbf{c}$ The relative expression of the ten indicated circRNAs listed in a from the chemoresistant cells and matched chemosensitive cells was measured by qRT-PCR; PDC1-S, patient-derived cells that are relatively sensitive to chemotherapy; PDC1-R, patient-derived cells that are relatively resistant to chemotherapy. Data are mean $\pm \mathrm{SD}, n=3$. d A schematic diagram of the genomic location and splicing pattern of cESRP1 is shown. e Random hexamer or oligo (dT)18 primers were used in reverse transcription experiments. The relative RNA levels were analysed by qRT-PCR and normalised to the level measured using the random hexamer primers. Data are mean $\pm \mathrm{SD}, n=3$. f The relative RNA levels of cESRP1 and mESRP1 in H69 and H446 cells were analysed by qRT-PCR after treatment with actinomycin D at the indicated time points. Data are mean $\pm \mathrm{SD}, n=3$. g cESRP1 and mESRP1 were abundant in the cytoplasm of $\mathrm{H} 69$ cells. $\beta$-actin and U6 were used as positive controls in the cytoplasm and nucleus, respectively. Data are mean $\pm \mathrm{SD}, n=3$. h RNA fluorescence in situ hybridisation for cESRP1 was performed in H446 cells. Nuclei were stained with 4,6-diamidino-2-phenylindole (DAPI). Scale bar, $5 \mu \mathrm{m}$

small $\left(1 \mathrm{~mm}^{3}\right)$ fragments and incubated for $1.5 \mathrm{~h}$ at $37^{\circ} \mathrm{C}$ in a phosphate buffer ( $\mathrm{pH} 7.0$ ) with $1 \mathrm{mg} / \mathrm{ml}$ of collagenase type I (Solarbio, Beijing, China), $1 \mathrm{mg} / \mathrm{ml}$ of collagenase type II (Solarbio, Beijing, China), and 50 units/ml DNase I (Sigma, MO, USA) with occasional vibration to achieve enzymatic disaggregation. Then, an equal volume of the phosphate buffer was added, after which the cells were resuspended by pipetting and then filtered through a 100- $\mu \mathrm{m}$ nylon mesh. Single cells were used for culture and cryopreservation.

\section{In vivo generation of acquired resistance to cisplatin/ etoposide $(C / E)$}

To establish a chemoresistant subcutaneous PDX model, we performed a previously described procedure [42] after the tumours grew to a size of $\sim 50 \mathrm{~mm}^{3}$. Cohorts of animals were treated once every ten days with cycles of cisplatin $(2.5 \mathrm{mg} / \mathrm{kg}$, i.p. injection on day 1$)$ and etoposide $(4 \mathrm{mg} / \mathrm{kg}$, i.p. injection on days 1-3), which were intraperitoneally injected at least six times during each round of treatment. When the tumour volume reached $\sim 500 \mathrm{~mm}^{3}$, the mice were euthanized, and the tumours were removed. The drugtreated tumours were divided into small fragments and engrafted into the next generation of mice following the same method of cisplatin/etoposide $(\mathrm{C} / \mathrm{E})$ or vehicle treatment until chemoresistant tumours developed.

\section{Chemosensitivity testing of the PDX model}

The chemotherapeutic response of the PDX model tumours was determined by digesting tumours into single-cell suspensions and subcutaneously injecting an equal number of cells into a large number of $\mathrm{B}-\mathrm{NDG}^{\circledR}$ mice. When the tumours reached a palpable size $(5 \mathrm{~mm})$, the mice were randomised into treatment and vehicle control groups. To examine the tumour response to first-line chemotherapeutic drugs for SCLC therapy, the following drugs and treatment modalities were used: cisplatin at a dosage of $2.5 \mathrm{mg} / \mathrm{kg} / \mathrm{d}$, qd 1, i.p. injection; and etoposide at a dosage of $4 \mathrm{mg} / \mathrm{kg} / \mathrm{d}$, qd 1-3, i.p. injection. Doses and schedules were chosen according to previous experience with animal studies and represent the maximum tolerated or efficient doses in our investigation.

To assess the sensitivity of tumours to chemotherapy after a combination treatment with chemotherapeutic drugs and the TGF- $\beta$ inhibitor LY2157299 (Selleck, Houston, TX, USA), the following treatment schedule was used: the vehicle control arm, $100 \mu \mathrm{l}$ of $0.9 \%$ saline administered intraperitoneally on days 1, 2, and 3; the LY2157299 arm, $4 \mathrm{mg} / \mathrm{kg}$ administered intraperitoneally on days 1,2 , and 3; the drug treatment arm, cisplatin $(2.5 \mathrm{mg} / \mathrm{kg})$ administered intraperitoneally on day 1 plus etoposide $(4 \mathrm{mg} / \mathrm{kg})$ administered intraperitoneally on days 1, 2, and 3; and the combination arm, LY2157299 (2 mg/kg) administered intraperitoneally on days 1,2 , and 3 plus cisplatin $(1.5 \mathrm{mg} / \mathrm{kg})$ administered intraperitoneally on day 1 and etoposide $(3 \mathrm{mg} / \mathrm{kg})$ administered intraperitoneally on days 1, 2, and 3. Animals were given a two-week respite from treatment to allow for weight recovery.

\section{Statistics and reproducibility}

All experiments were performed independently three or more times under similar conditions. The results are presented as the means, and the error bars represent the standard deviation (SD) unless stated otherwise. Prism 5.0 was used to generate graphs, and SPSS 20.0 was used to perform statistical analyses, with $p$ values calculated using the chi-square test, one-way ANOVA or unpaired two-tailed Student's $t$ test. Survival durations were analysed using the Kaplan-Meier method and were compared among groups using the log-rank test. Hazard ratios were calculated using a Cox-regression analysis. Significant $p$ values are represented as $* p \leq 0.05, * * p \leq 0.01, * * * p<0.001$, and n.s. no significance.

\section{Results}

\section{Deregulated circRNAs in chemoresistant SCLC and CESRP1 characterisation}

We first analysed the circRNA expression profiles of SCLC cells by using a circRNA microarray (Fig. S1a; 

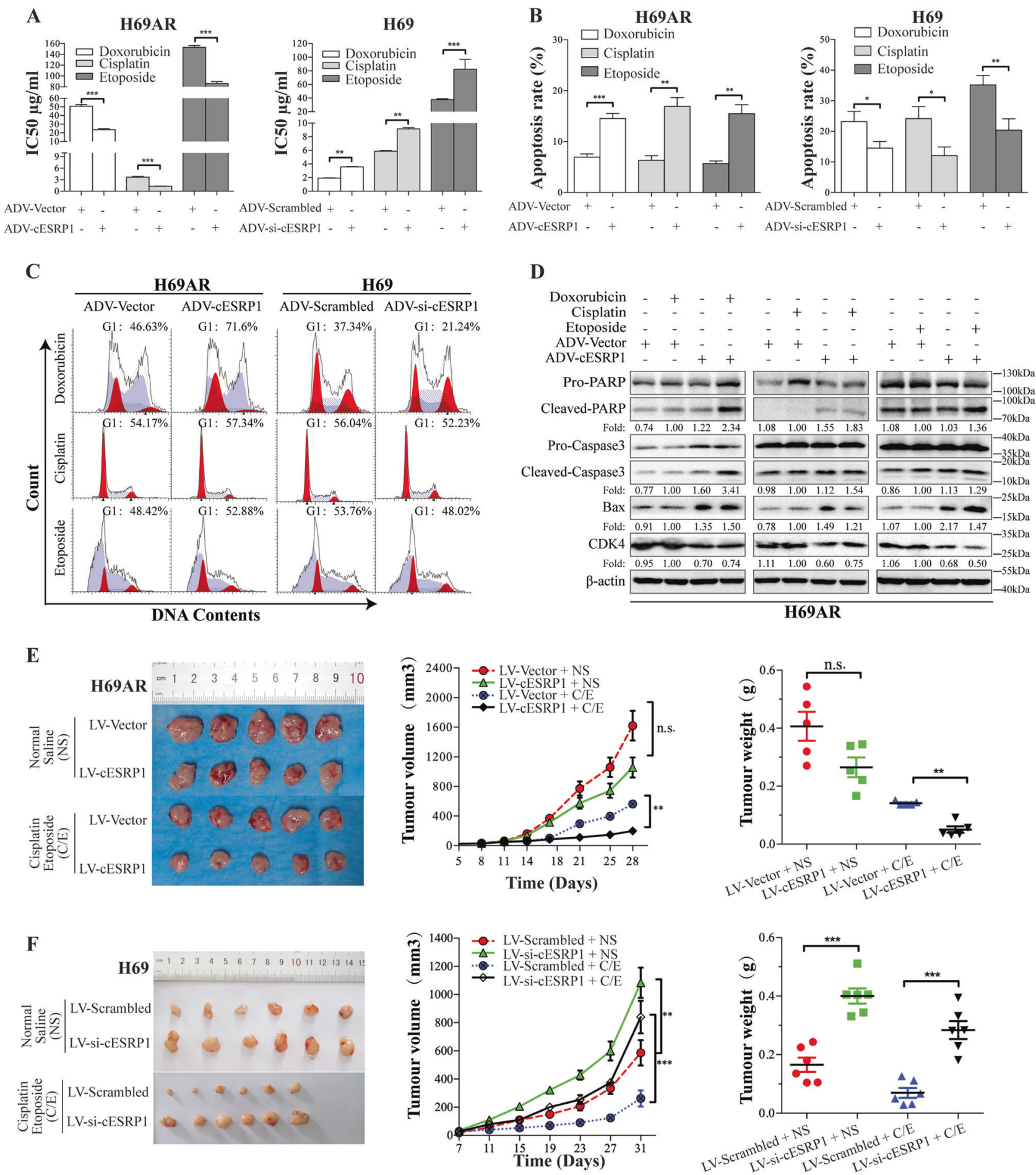

Fig. 2 cESRP1 inhibits SCLC chemoresistance. a Bar graph representing the effect of cESRP1 modulation on the drug sensitivity of SCLC cells measured by a CCK8 assay. Data were pooled from four biological replicates $\pm \mathrm{SD}, n=4$. b Bar graph showing the effect of cESRP1 modulation on the cell apoptosis rate after chemotherapy treatment in SCLC cells. Data are mean $\pm \mathrm{SD}, n=3$. c FACS analysis showing an increase in the proportion of cells in the G1 phase in chemoresistant cells overexpressing cESRP1 and treated with chemotherapeutic drugs, whereas a decrease in the G1 population was observed when cESRP1 was silenced in chemosensitive cells. d PARP, caspase3, Bax, and CDK4 expression levels detected by western blot analysis in cESRP1overexpressing H69AR cells following treatment with chemotherapeutic drugs. e Effects of cESRP1 overexpression on tumour growth in vivo. Left: Representative images of tumours formed in nude mice after subcutaneous injection of cESRP1-overexpressing H69AR cells are shown $(n=5)$. Middle: Tumours comprising cESRP1-overexpressing H69AR cells showed markedly lower growth rates than those comprising control cells. Right: Tumour weight was measured. Data are mean \pm SD. f Effects of cESRP1 expression knockdown on tumour growth in vivo. Left: Representative images of tumours formed in nude mice from subcutaneously injected cESRP1-knockdown H69 cells $(n=6)$. Middle: Tumour growth curves. Tumours comprising cESRP1-knockdown cells showed markedly higher growth rates than control cells. Right: Tumour weight was measured. Data are mean $\pm \mathrm{SD}$ 


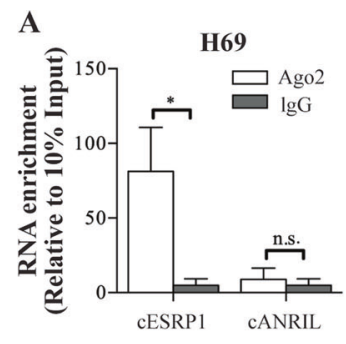

C

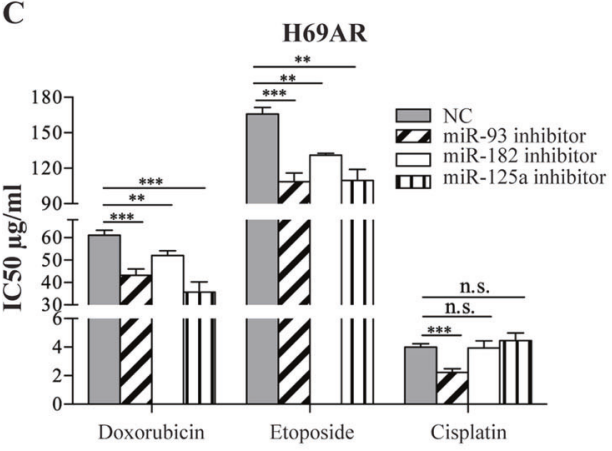

F
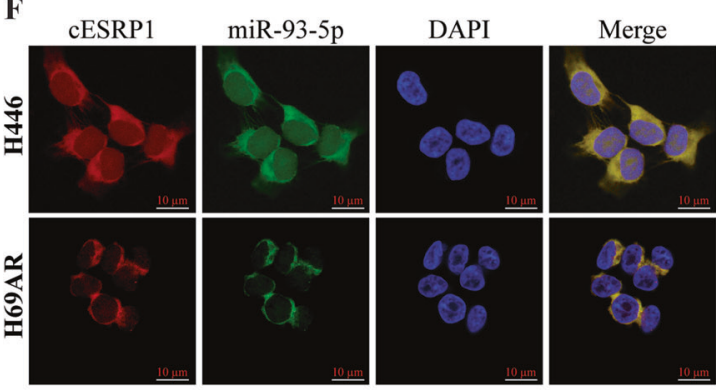

$\mathbf{H}$

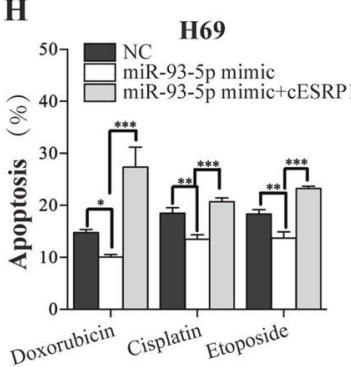

I
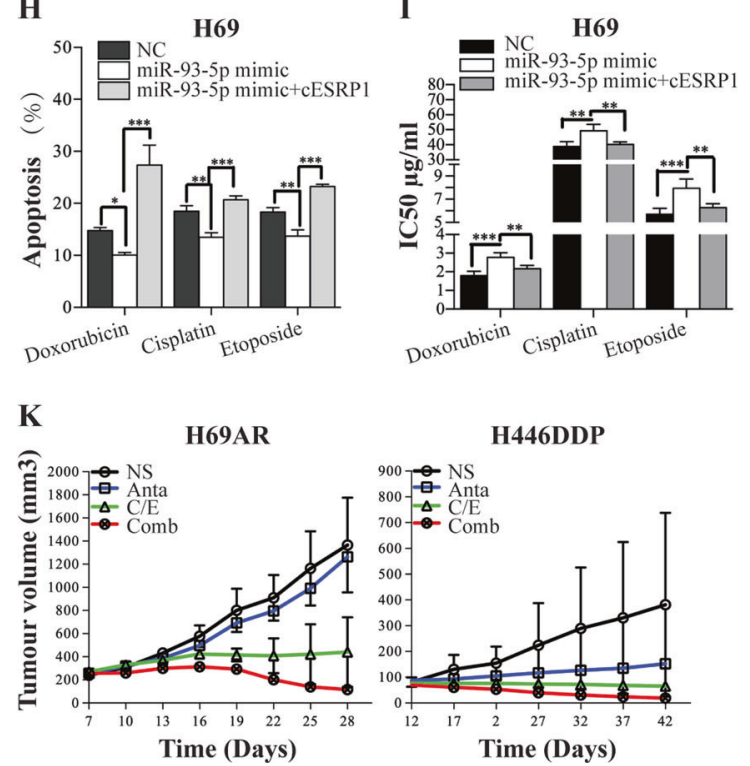

Supplementary Table 1). The expression analysis results identified 224 differentially expressed circRNAs, with 138 circRNAs exhibiting upregulated expression and 86
B
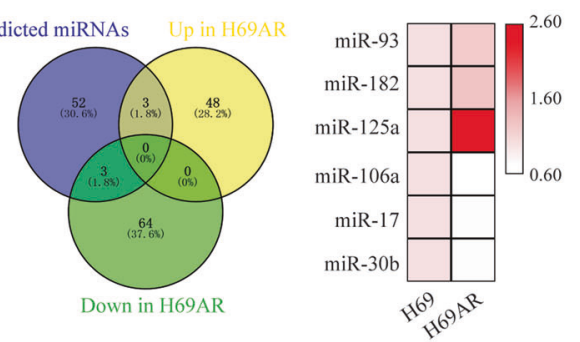

E

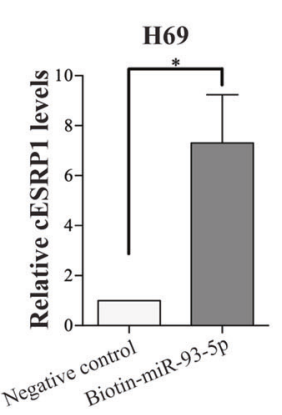
cESRP1-WT (107-129): 5' - gccaagGGAGGTGCAGCACT T Tg - 3, hsa-miR-93-5p: 3'- gauggaCGUGCUUGUCGUGAAAc - 5, cESRP1-MUT (107-129): 5' - gccaagGGAGGTGCAG CTAGGCg - 3'
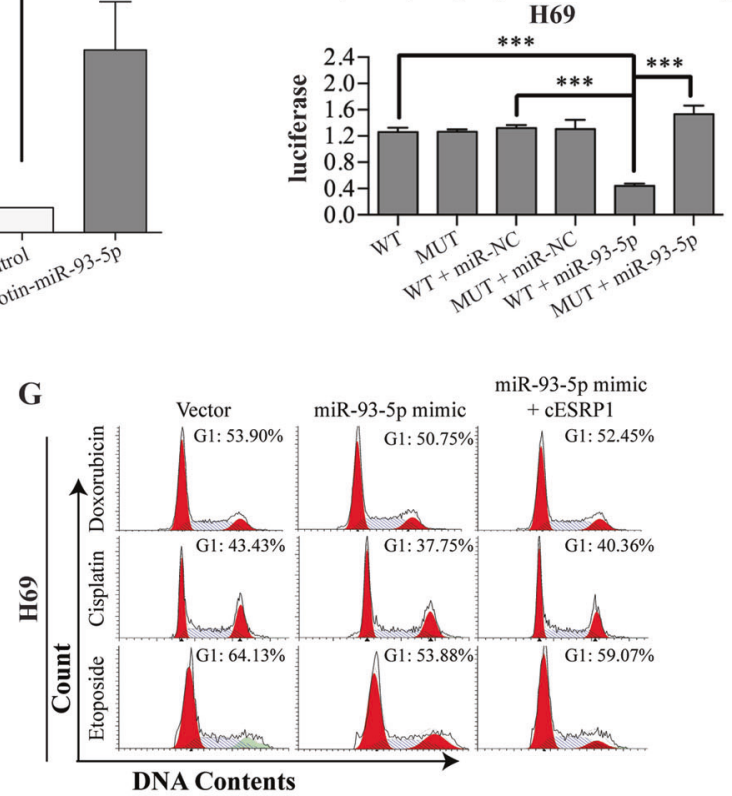

J

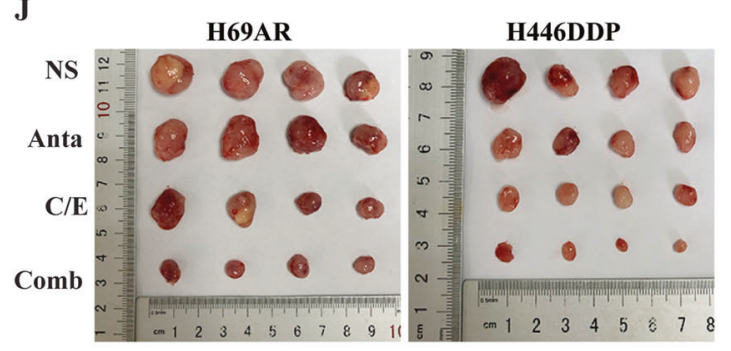

L

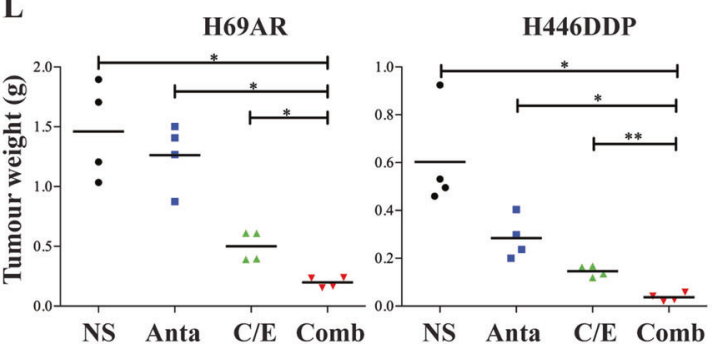

exhibiting downregulated expression in H69AR as compared with H69. Ten circRNAs with the greatest increases and decreases in expression are shown in Fig. 1a. 
Fig. 3 cESRP1 functions as a sponge of miR-93-5p in SCLC. a RIP experiments were performed using an antibody against $\mathrm{AGO} 2$ with extracts from SCLC cells. Data are mean $\pm \mathrm{SD}, n=3$. b The Venn diagram shows the intersection of miRNA lists. The heatmap shows the expression of the overlapping miRNAs in chemoresistant and chemosensitive SCLC cell lines. c The bar graph shows the effect of miR-93-5p, miR-182-5p, and miR-125a-5p inhibition on the drug sensitivity of H69AR cells. Data were pooled from four biological replicates $\pm \mathrm{SD}, n=4$. d qRT-PCR was used to analyse the cESRP1 levels in streptavidin-captured fractions from H69 cell lysates after transfection with $3^{\prime}$-end biotinylated miR-93-5p or a negative control. Data are mean $\pm \mathrm{SD}, n=3$. e H69 cells were co-transfected with LUCcESRP1-WT or LUC-cESRP1-MUT vectors and an miR-93-5p mimic or a negative control (miR-NC). Luciferase activity was detected with luciferase reporter assays. Data are mean $\pm \mathrm{SD}, n=3$. f The colocalization of cESRP1 and miR-93-5p was observed by RNA in situ hybridisation in H446 and H69AR cells. Nuclei were stained with a DAPI solution. $\mathbf{g}-\mathbf{h}$ Cell cycle and cell apoptosis rate analyses of H69 cells that received the indicated treatments are shown. Data are mean $\pm \mathrm{SD}, n=3$. i The IC50 values of H69 cells transfected with the indicated transcripts and treated with drugs were measured using CCK-8 assays. Data are mean $\pm \mathrm{SD}, n=4$. $\mathbf{j}$ Images of subcutaneous tumours comprising H69AR or H446DDP cells after cisplatin/etoposide $(\mathrm{C} / \mathrm{E})$ treatment or combination treatment $(\mathrm{Comb})$ with the miR93-5p antagomir (Anta) are shown $(n=4)$. NS normal saline. $\mathbf{k}$ The growth curves of xenografted tumours derived from SCLC cells with or without miR-93-5p antagomir or cisplatin/etoposide treatment are shown. Data are mean $\pm \mathrm{SD}, n=4$. 1 Tumour weight (means) was measured at the endpoint

We verified part of circRNA microarray results by qRTPCR in H69AR/H69 cells as well as in the other two compared cells H446DDP/H446 and PDC1-R/PDC1-S. We validated five upregulated and five downregulated circRNAs observed in H69AR cells using circRNA-specific divergent primers and Sanger sequencing (Fig. 1b; Fig. S1b). Furthermore, these ten circRNAs were proven to be real circRNAs after treatment with RNase R (Fig. S1c). Of the ten circRNAs, we observed six circRNAs (cESRP1, hsa_circ_0084927; cNRIP1, hsa_circ_0061276; cMPPED2, hsa_circ_0021553; cNFIX, hsa_circ_0049657; cCRIM1, hsa_circ_0005579; and cEPB41, hsa_circ_0000042) with consistent and significant deregulated expression in chemorensistant cells (Fig. 1c). Among these circRNAs, because cESRP1 originates from the ESRP1 gene, which is closely linked to tumour-associated EMT [45, 46], we selected cESRP1 to further investigate the role of cESRP1 in SCLC chemoresistance.

cESRP1 is produced from exons 7, 8, and 9 of the ESRP1 gene locus (CircBase ID: hsa_circ_0084927, referred to as cESRP1, http://www.circbase.org/cgi-bin/listsea rch.cgi) (Fig. 1d). To confirm the characteristics of cESRP1, we used random hexamer or oligo (dT)18 primers in reverse transcription experiments. Compared with the results obtained using the random hexamer primers, the relative expression of cESRP1 was observed to be significantly downregulated using the oligo (dT)18 primers, whereas the linear RNA mESRP1 RNA was not (Fig. 1e). This finding suggested that cESRP1 did not have a poly-A tail. Furthermore, actinomycin D was used to inhibit transcription and compare the half-lives of cESRP1 and mESRP1, the results of which showed that cESRP1 was more stable than mESRP1 in SCLC cells (Fig. 1f). We also presented cESRP1 was primarily localised in the cytoplasm in SCLC cells by qRT-PCR and FISH (Fig. 1g, h).

\section{CESRP1 inhibits SCLC chemoresistance in vitro and in vivo}

We further investigated the function of cESRP1 in SCLC chemoresistance by first constructing transient cESRP1overexpressing cells (ADV-cESRP1) and cESRP1knockdown cells (ADV-si-cESRP1) using adenoviral vectors in chemoresistant and chemosensitive cells, respectively (Fig. S2a). Subsequently, cell viability, cell cycle distribution, and cell apoptosis were examined after treatment with chemotherapeutic drugs, including doxorubicin, cisplatin, and etoposide. The results indicated that cESRP1 upregulation promoted cell chemosensitivity, with a significant decrease in the IC50 value observed as well as increases in cell apoptosis and G0/G1 cell arrest, whereas reduced cESRP1 expression led to the opposite results (Fig. 2a-c; Fig. S2b-d). Immunoblotting was performed to assess proteins levels in apoptotic and cell cycle pathways. Notably, the immunoblotting results showed that apoptotic protein markers, including cleaved PARP, cleaved caspase3, and Bax, increased after cESRP1 overexpression in chemoresistant cells. We also observed that the level of the G1/S phase checkpoint protein CDK4 decreased after treatment with doxorubicin, cisplatin, or etoposide in the cESRP1-overexpressing cells compared with the vectortransfected cells (Fig. 2d; Fig. S2e).

We next tested the effect of cESRP1 on tumour chemotherapeutic reactivity in vivo. We constructed cell lines stably overexpressing or underexpressing cESRP1 (H69AR-LV-cESRP1 and H69-LV-si-cESRP1, respectively) using lentiviral vectors. We then performed a tumourigenesis assay by subcutaneously injecting cESRP1overexpressing cells or control cells into the flanks of nude mice. The results showed that tumour growth was slowed by cESRP1 overexpression in both the normal saline (NS) cohort and the C/E-treated cohort (Fig. 2e; Fig. S2f). In contrast, we observed that tumour growth was accelerated by cESRP1 knockdown (Fig. 2f). Together, these data indicated that cESRP1 rescues SCLC chemoresistance.

\section{CESRP1 may function as a sponge of miR-93-5p in SCLC}

It is accepted that circRNAs can function as sponges of miRNAs and that cESRP1 is stable and located in the 

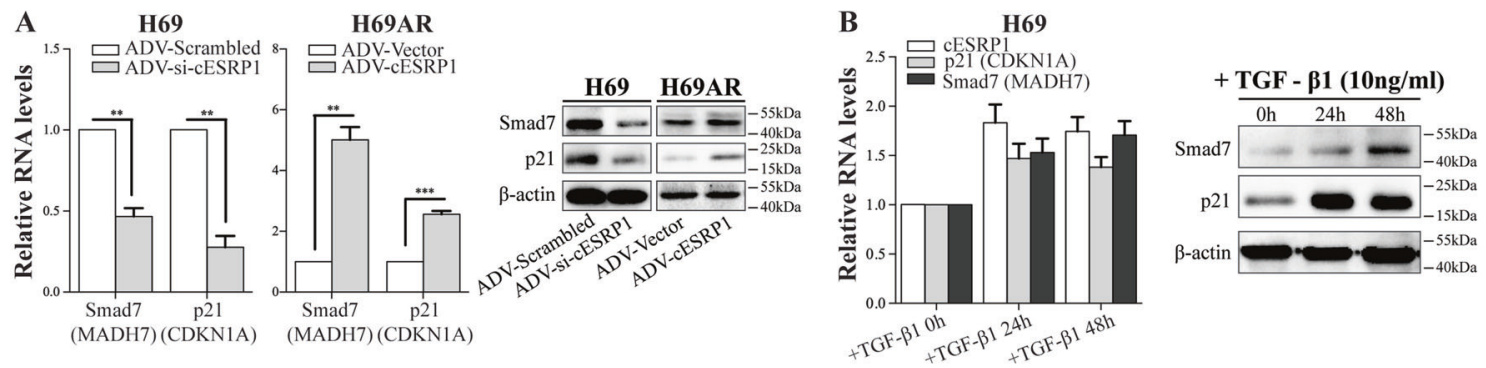

C

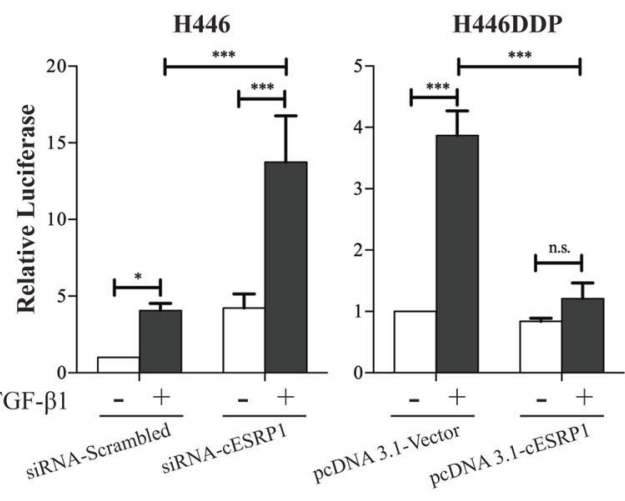

E

$\begin{array}{lllllllll}\mathrm{ADV}-S c r a m b l e d & + & + & - & - & + & + & - & - \\ \mathrm{ADV}-\mathrm{si} \text {-cESRP1 } & - & - & + & + & - & - & + & +\end{array}$ miR-93-5p inhibitor

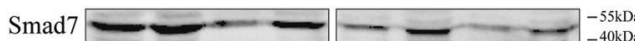
\begin{tabular}{llllllll} 
Fold: 1.00 & 1.16 & 0.65 & 0.89 & 1.00 & 1.13 & 0.69 & 0.92 \\
\hline
\end{tabular} $\mathrm{p} 21-0-25-25 \mathrm{ka}$

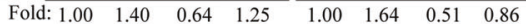

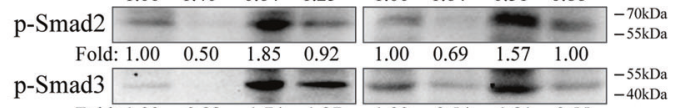

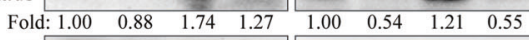
Total-Sma 2 Total-Smad3

Smad4

E-cad

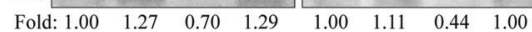

$\beta$-actin -

H69

\begin{tabular}{c} 
H69AR \\
\cline { 2 - 2 } ADV-Vector \\
\hline
\end{tabular}

\begin{tabular}{rlllllllll} 
TGF $-\beta 1$ & - & - & + & + & - & - & + & + \\
miR-93-5p mimic & - & + & - & + & - & + & - & + \\
\hline
\end{tabular}

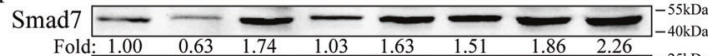
$\mathrm{p} 21 \div-\frac{-2-2.26}{-25 \mathrm{kDa}}$ Fold: \begin{tabular}{llllllll}
1.00 & 0.56 & 1.52 & 1.34 & 1.75 & 1.39 & 1.90 & 1.94 \\
\hline & & & & & & &
\end{tabular} p-Smad2

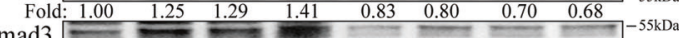

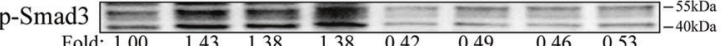
Total-Smad2 - - - - - - - $-70 \mathrm{kDa}$ Total-Smad3 Smad4

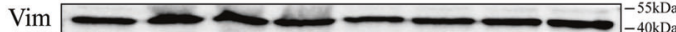

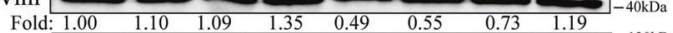

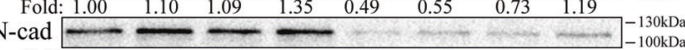

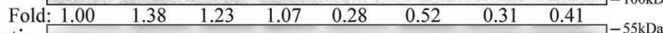
$\beta$-actin $\longrightarrow$

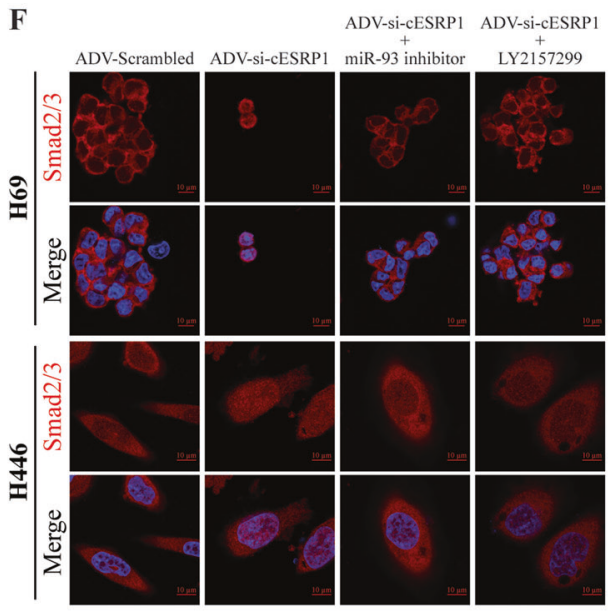

G

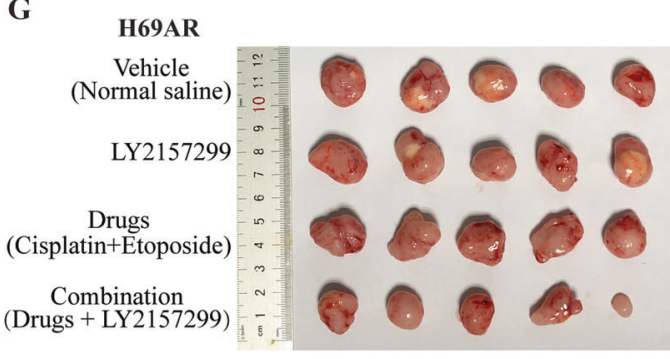

cytoplasm of SCLC cells; thus, exploring whether cESRP1 can bind to miRNAs may be valuable. We first conducted an RIP assay with an antibody against AGO2 in SCLC
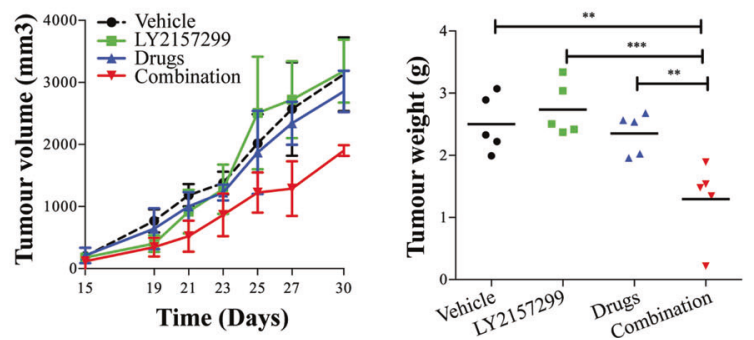

cells. The results showed that cESRP1 but not the negative control cANRIL [47], was significantly enriched using the anti-AGO2 antibody (Fig. 3a). This result suggested that 
Fig. 4 cESRP1 prohibits TGF- $\beta$-mediated EMT via the miR-93-5pSmad7/p21(CDKN1A) axis. a Smad7 and p21(CDKN1A) expression were analysed after interfering with cESRP1 expression in SCLC cells. Data are mean $\pm \mathrm{SD}, n=3$. b The transcriptional or translational levels of cESRP1, Smad7, and p21(CDKN1A) were upregulated after treatment of $\mathrm{H} 69$ cells with TGF- $\beta 1$. Data are mean $\pm \mathrm{SD}, n=3$. c SCLC cells were co-transfected with a CAGA-luciferase reporter and cESRP1-siRNA vector or cESRP1-overexpressing vector. The cells were stimulated with TGF- $\beta 1$ overnight, and luciferase activity was measured. A scrambled vector or an empty vector was used as a control. The data are presented as the means of triplicate samples from a representative experiment performed three times \pm SD. d Western blotting was used to analyse cESRP1-overexpressing H69AR cells treated with TGF- $\beta 1(10 \mathrm{ng} / \mathrm{ml})$ or transiently transfected with an miR93-5p mimic. e Western blot analysis of SCLC chemosensitive cells transiently transfected with a miR-93-5p inhibitor after the transient knockdown of cESRP1 expression by an adenovirus. f Immunofluorescence staining shows intracellular $\operatorname{Smad} 2 / 3$ in the indicated cells. g A subcutaneous implantation model was established using H69AR cells. The graphs below show the tumours that developed and a statistical plot of the tumour growth kinetics (means \pm SD) as well as the average tumour weights in the subcutaneous implantation model mice. $n=5$

cESRP1 may act as an miRNA sponge to be involved in the AGO2-mediated ceRNA mechanism of action.

We next examined potential miRNAs associated with cESRP1 using the miRcode and TargetScan prediction tools (Supplementary Table 2). According to our miRNA microarray [48] and the above-predicted miRNAs, three miRNAs (miR-93, miR-182, miR-125a) were up-expressed, and three (miR-106a, miR-17, miR-30b) down-expressed in H69AR cells compared with H69 cells (Fig. 3b; Supplementary Table 3). However, the expression of these six miRNAs was upregulated in chemoresistant cells compared with chemosensitive cells, as confirmed by qRT-PCR assays (Fig. S3a). Subsequently, we observed that the enrichment of these six miRNAs was unaffected by the cESRP1 levels in SCLC cells (Fig. S3b). These findings suggest that miRNAs may not be degraded by cESRP1, as previously described [47]. Based on the results of previous studies [49-52], we focused on miR-93-5p, miR-182-5p, and miR-125a-5p and further investigated their roles in chemoresistance in SCLC cells. Using specific miRNA inhibitors, we successfully downregulated the expression of these three miRNAs in SCLC cells (Fig. S3c) and then treated the cells with chemotherapeutic drugs. We observed that the inhibition of miR-182-5p, miR-93-5p, and miR$125 \mathrm{a}-5 \mathrm{p}$ promoted chemosensitivity to doxorubicin and etoposide. In addition, miR-93-5p knockdown also facilitated chemosensitivity to cisplatin (Fig. 3c). Thus, we selected miR-93-5p for the follow-up studies. To identify whether miR-93-5p can bind to cESRP1, we purified miR93-5p-associated RNAs by biotin-miRNA pulldown and observed a 7.3-fold enrichment in cESRP1 in the biotinylated miR-93-5p-captured fraction compared with the negative control fraction (Fig. 3d). To further determine whether cESRP1 harbours an miR-93-5p binding domain, we constructed a luciferase reporter gene vector containing either the WT or mutant (MUT) cESRP1 and then cotransfected miR-93-5p mimics with the luciferase reporter gene vectors into $\mathrm{H} 69$ and $\mathrm{H} 446$ cells. Compared with a negative control RNA, the miR-93-5p mimic reduced the luciferase reporter activity in SCLC cells transfected with the WT cESRP1 construct (Fig. 3e; Fig. S3d). In addition, double FISH indicated the colocalization of cESRP1 and miR-93-5p in H446 and H69AR cells by fluorescence confocal microscopy (Fig. 3f).

Subsequently, we further assessed the effects of cESRP1 on the function of miR-93-5p with respect to drug resistance in SCLC. Our results showed that miR-93-5p overexpression significantly decreased the G0/G1 cell proportion and cell apoptosis but markedly enhanced cell viability in response to drug treatment, whereas reintroducing exogenous cESRP1 abolished the drug resistance-promoting effects of miR-93-5p on SCLC cells in vitro (Fig. 3g-i; Fig. S3e-f). Notably, intravenous administration of a cholesterolmodified miR-93-5p antagomir markedly enhanced the sensitivity of tumours to chemotherapeutic drugs in vivo (Fig. 3j-1). The results of these experiments indicate that cESRP1 may function as a sponge of miR-93-5p in SCLC.

\section{CESRP1 prohibits TGF- $\beta$-mediated EMT via the miR- 93-5p-Smad7/p21(CDKN1A) axis in SCLC}

Previous reports have demonstrated that the miR-106b-25 cluster (miR-106b, miR-93, and miR-25), which is activated by $\mathrm{E} 2 \mathrm{~F} 1$, can inhibit the growth-suppressing functions of TGF- $\beta$ signalling by interfering with the downstream mediators p21 (CDKN1A, Waf1/Cip1) and Bim (BCL2L11) in gastric cancer [53]. These miRNAs can also target the TGF- $\beta$ inhibitor Smad7 to activate the TGF- $\beta$ signalling and induce EMT and tumour initiating cell characteristics downstream of Six 1 in human breast cancer [54]. Based on these findings, we hypothesised that cESRP1 inhibits chemoresistance in SCLC by protecting Smad7 and p21(CDKN1A) from downregulation by miR-93-5p, thus inhibiting the tumour-promoting functions of TGF- $\beta$ signalling. To test this hypothesis, we first examined the expression of Smad7 and p21(CDKN1A) in chemosensitive and chemoresistant SCLC cells, both of which were observed to be significantly downregulated in the chemoresistant cells (Fig. S4a). Second, we found the Smad7 and p21(CDKN1A) levels were significantly downregulated upon miR-93-5p overexpression, whereas they were significantly upregulated when miR-93-5p knockdown in SCLC cells (Fig. S4b). Third, we demonstrated that the Smad7/p21(CDKN1A) levels were strikingly upregulated when cESRP1 was overexpressed and downregulated when cESRP1 expression was knocked down (Fig. 4a; Fig. S4c). 


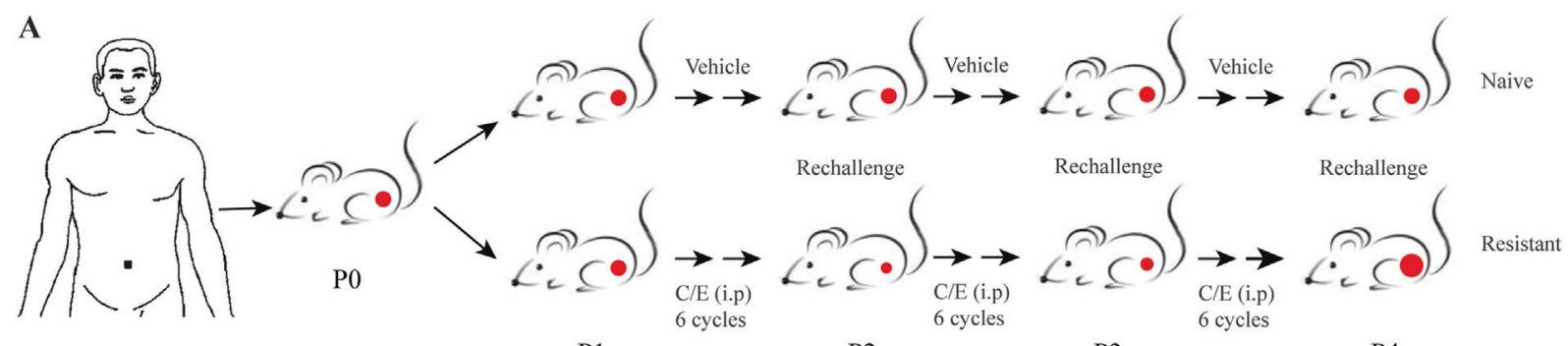

$\begin{array}{llll}\text { P1 } & \text { P2 } & \text { P3 } & \text { P4 }\end{array}$

B Resistant-Vehicle

Resistant-C/E PDX1

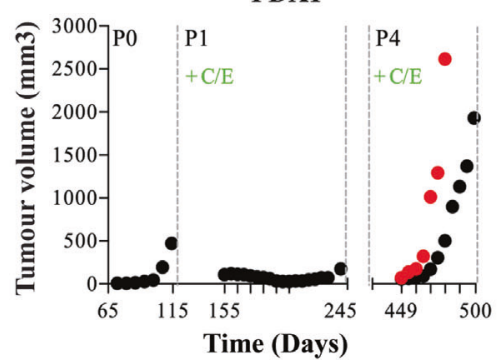

PDX2

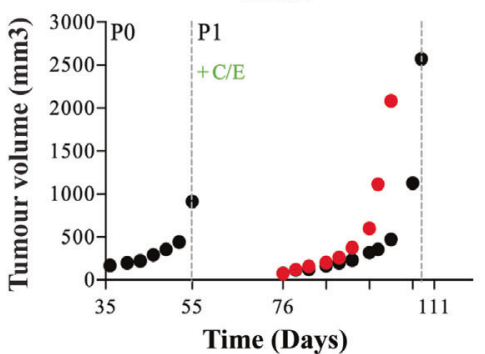

C

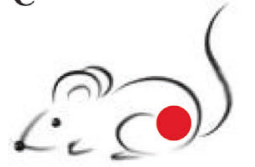

$$
\downarrow \begin{gathered}
\text { Fresh xenograft } \\
\text { tumour tissue }
\end{gathered}
$$
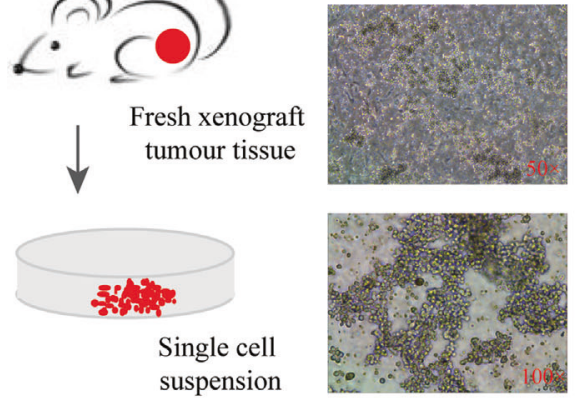

D

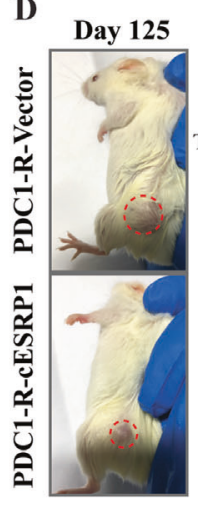

PDX3

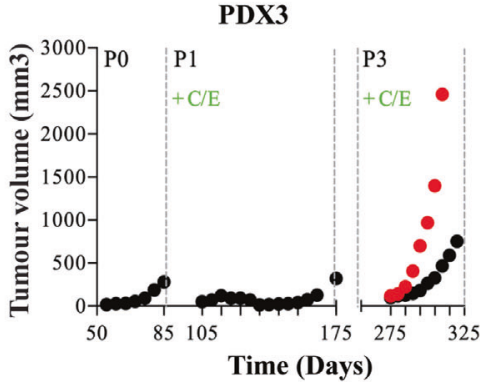

Time (Days)
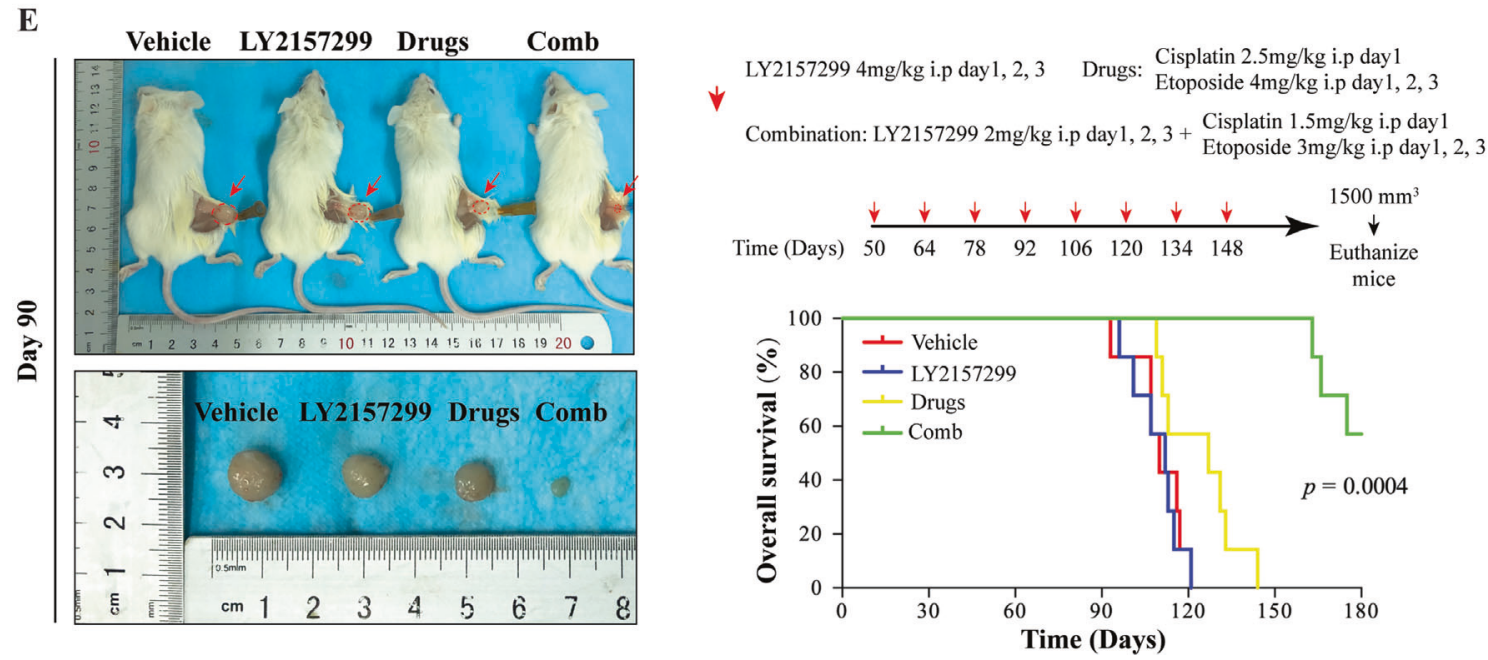

More interestingly, with the increase in exogenous cESRP1 expression, the expression of Smad7/p21(CDKN1A) was less inhibited by the exogenous miR-93-5p mimic
(Fig. S4d). These data indicated that miR-93-5p and cESRP1 efficiently interfered with Smad7/p21(CDKN1A) expression in SCLC. 
Fig. 5 TGF- $\beta$ signalling inhibition augments the chemoresponsiveness of SCLC PDX. a Outline of the experimental approach, beginning with the primary sample. b Tumour growth kinetics of PDX1, PDX2, and PDX3 models. Average tumour volumes $(n=10)$ of different generations of xenograft tumours are shown (P0 was the initial xenograft tumour and was not exposed to C/E; P1, P2, P3, and P4 represent the second, third, fourth, and fifth generation re-engrafted tumours, which were treated with $\mathrm{C} / \mathrm{E}$ (black dots) or a vehicle (red dots)). The ticks on the $x$-axis indicate day 1 of every $\mathrm{C} / \mathrm{E}$ cycle. The dashed vertical lines and $x$-axis days indicate the times at which the treated tumours were collected and re-engrafted into the next cohort. $\mathbf{c}$ Digestion of xenograft tumour tissue into single-cell suspensions for in vitro culture. d Photograph of mouse tumours on day 125 after implantation of PDC1-R-Vector cells or PDC1-R-cESRP1 cells treated with chemotherapeutic drugs (Left). The overall survival (OS) of the mice in the two indicated groups is shown (Right) $(n=7)$. OS: Time until the tumour volume reached $1500 \mathrm{~mm}^{3}$. e Representative image of tumours derived from mice on day 90 after re-engraftment of PDX1P4-resistant tumour tissue (Left) and the OS of the experimental PDX model mice receiving the indicated treatments shown (Right) $(n=7)$. The TGF- $\beta$ signalling inhibitor LY2157299, when administered in combination with chemotherapeutic drugs, exhibited remarkable inhibitory effects on tumour growth, whereas LY2157299 treatment alone did not display a therapeutic impact

Many studies have shown that TGF- $\beta$ can serve as a tumour suppressors to induce the expression of Smad7 and p21(CDKN1A) in certain cancers [55-57]. Interestingly, Smad7 and p21(CDKN1A) may act as components in a negative feedback regulation of TGF- $\beta$ signalling, as Smad7 impedes TGF- $\beta /$ Smad-driven transcription $[21,58,59]$. Although p21(CDKN1A) is a direct target of Smad2/3 [60], it could repress features of EMT by non-canonical TGF- $\beta$ pathways such as c-Myc and TGF $/$ MEK/ERK signalling $[31,32]$. To investigate whether cESRP1 is also involved in the negative regulation of TGF- $\beta$ signalling in SCLC, we first treated chemosensitive cells with TGF- $\beta 1$ (Fig. S4e) and observed that the TGF- $\beta 1$ treatment efficiently increased the expression of cESRP1, Smad7, and p21 (CDKN1A) (Fig. 4b; Fig. S4f). These results are consistent with those of previous studies [18, 61, 62]. Furthermore, our data revealed that the upregulation of cESRP1 by TGF- $\beta 1$ is independent of changes in the parental mESRP1 transcript (Fig. S4g). We then asked whether cESRP1 is a relevant factor in the regulation of TGF- $\beta$ activity in SCLC. We observed that silencing cESRP1 greatly enhanced the CAGA-luciferase activity of a TGF- $\beta$-responsive reporter, whereas ectopic expression of cESRP1 weakened CAGAluciferase activity (Fig. 4c). Furthermore, the transient overexpression of cESRP1 resulted in a decrease in the protein levels of $\mathrm{p}-\mathrm{Smad} 2 / 3$ and the mesenchymal markers $\mathrm{N}$-cadherin and vimentin, while treatment with a miR-93-5p mimic could partly block cESRP1-induced TGF- $\beta / S m a d$ signalling inhibition (Fig. 4d; Fig. S4h). In contrast, cESRP1 silencing enhanced TGF- $\beta$-Smad2/3 pathway activity, as evidenced by increased levels of $\mathrm{p}-\mathrm{Smad} 2 / 3$ and decreased expression of the epithelial marker E-cadherin, whereas downregulating miR-93-5p expression reversed the effect of cESRP1-siRNA (Fig. 4e). Importantly, Smad2/3 predominantly accumulated in the nucleus of cESRP1silenced cells, while knocking down miR-93-5p expression or treating cells with the TGF- $\beta$ signalling inhibitor LY2157299 reversed the nuclear translocation of Smads induced by cESRP1 silencing (Fig. 4f). The miR-93-5p mimic and TGF- $\beta 1$ could both rescue the cESRP1-mediated effect on the cytoplasmic localisation of Smad2/3 in the SCLC cells (Fig. S4i).

To further investigate the functional links between TGF$\beta$ signalling and drug resistance in vivo, we treated SCLC chemoresistant cell-derived xenograft mice with either vehicle or the TGF- $\beta$ signalling inhibitor LY2157299 in conjunction with chemotherapy drugs (C/E). Vehicletreated tumours and LY2157299-treated tumours expanded quickly, whereas a significant decline in tumour growth was observed when the chemotherapy drugs $\mathrm{C} / \mathrm{E}$ were combined with a TGF- $\beta$ inhibitor (Fig. 4g). These data underscore that TGF- $\beta$ inhibition significantly enhances the responsiveness to treatment in SCLC.

\section{TGF- $\beta$ signalling inhibition augments the chemoresponsiveness of SCLC patient-derived xenografts}

We next utilised SCLC PDX to further understand the effects of TGF- $\beta$ signalling on SCLC. We treated tumourbearing mice with repeated chemotherapy cycles that mimicked clinical practice in three independent SCLC PDX models (Fig. 5a; Supplementary Table 4). Histological comparison of the founder (P0) PDX tumours showed strong similarities to corresponding patient tumour samples (Fig. S5a). With multiple cycles of chemotherapy, we observed that PDX1 developed chemoresistance in the fourth round of $\mathrm{C} / \mathrm{E}$ treatment and that PDX3 acquired chemoresistance in the third round, while the PDX2 model showed initial chemoresistance without any dose-limiting toxicity as measured by animal weight (Fig. 5b; Fig. S5b).

We established one cell line from fresh drug-resistant PDX1 (PDC1-R) xenograft tumour tissue as described (Fig. 5c). We then stably overexpressed cESRP1 in the PDC1-R cells (Fig. S5c). A subcutaneous xenograft model established with the PDC1-R cells showed that the overall survival (OS) of mice bearing a tumour comprising cESRP1-overexpressing cells was significantly better than that of mice bearing the corresponding control tumour that developed from empty vector-infected PDC1-R cells (Fig. 5d). We also further verified that TGF- $\beta$ inhibitor significantly enhanced the tumour responsiveness to treatment in SCLC PDX (Fig. 5e). 


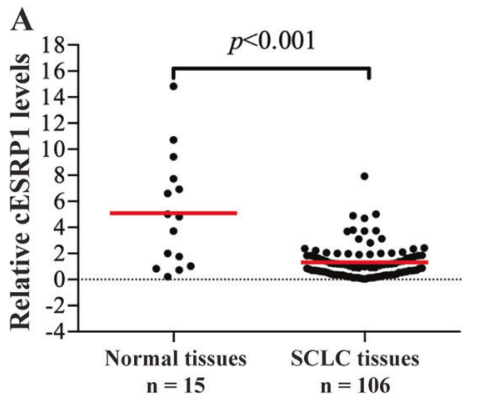

C

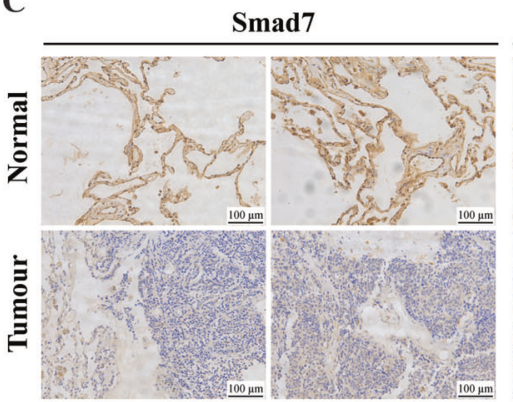

B
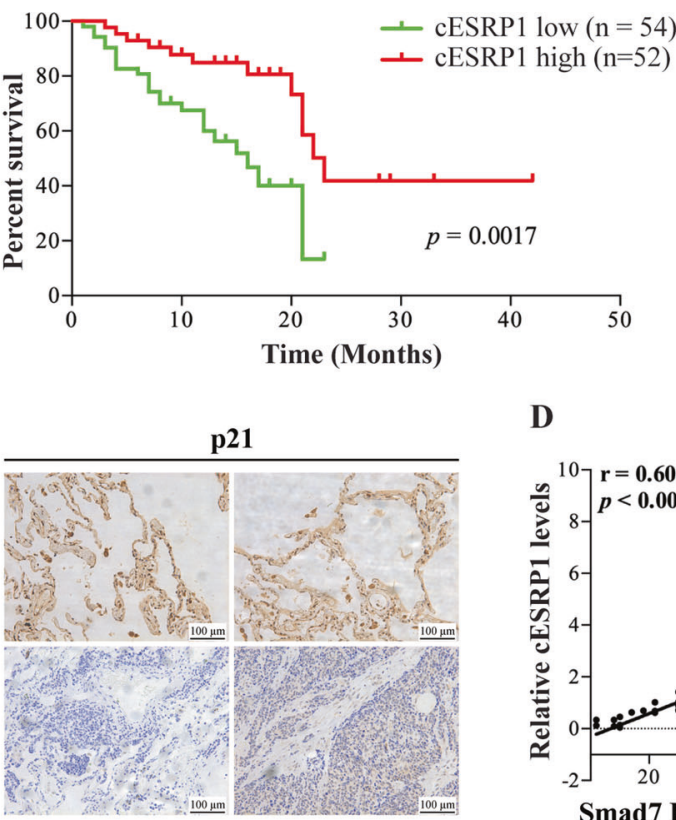

D
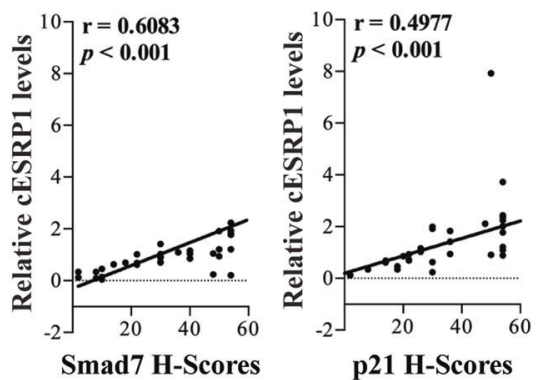
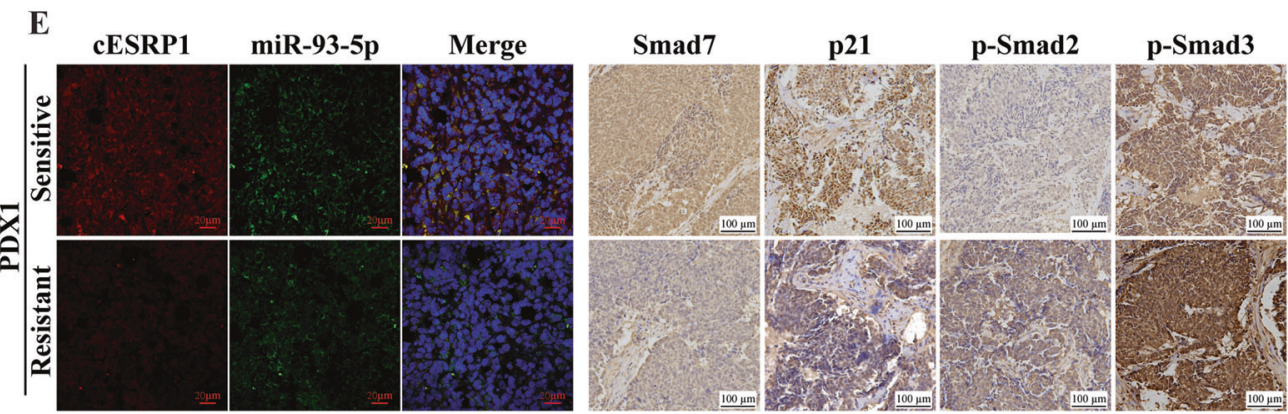

E-cad

Vim

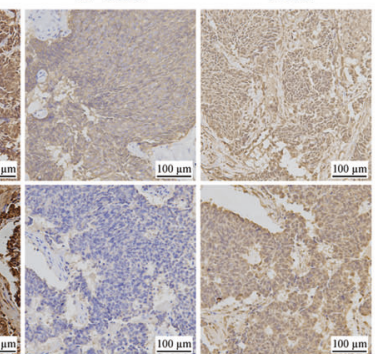

F

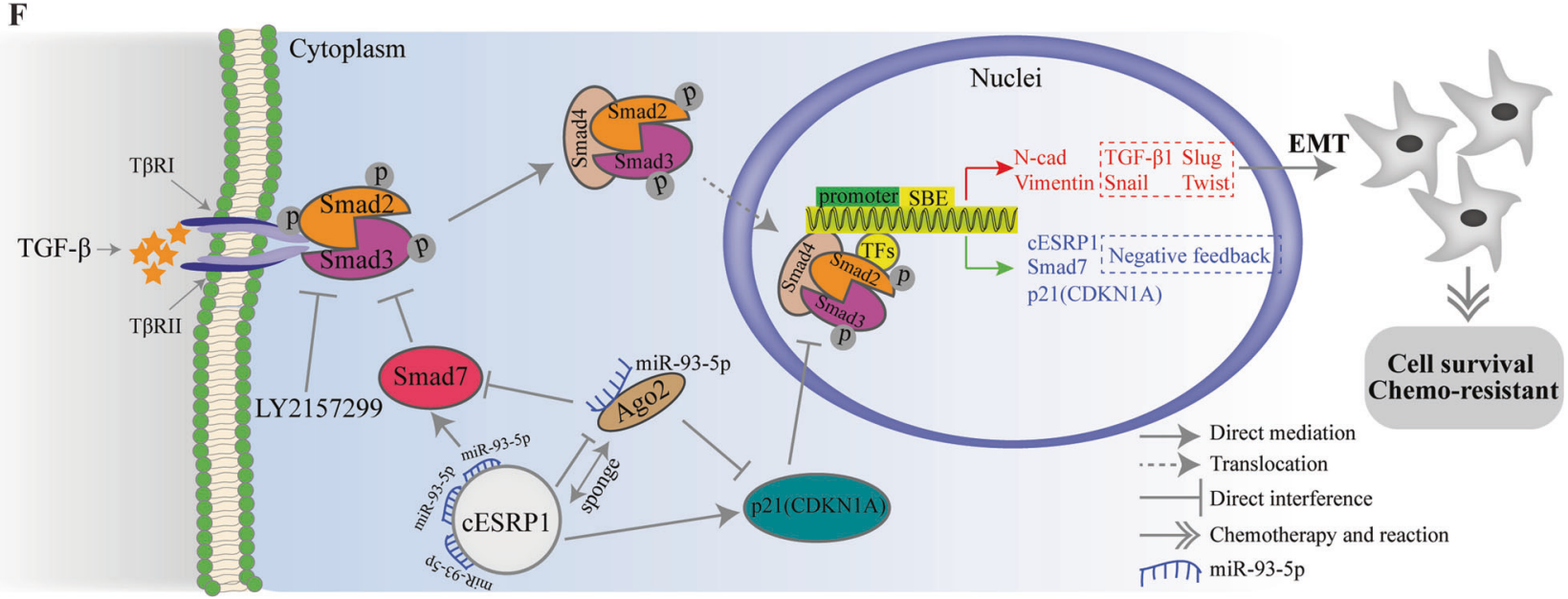

Fig. 6 cESRP1 expression is downregulated in SCLC tissues and low expression of cESRP1 predicts a poor prognosis. a The differential expression of cESRP1 between SCLC tissue samples and matched normal tissue samples was assessed. The median cESRP1 expression level of each group is indicated by a horizontal line in the scatterplot. b Kaplan-Meier survival curves show the correlations between cESRP1 expression and overall survival in SCLC patients. c Immunohistochemical staining for Smad7/p21 in SCLC tissue samples and adjacent normal lung tissue samples is shown. $\mathbf{d}$ The expression levels of Smad7/p21 positively correlated with those of cESRP1 in SCLC tissue samples $(n=48)$. e cESRP1 expression was assessed in situ by an RNA FISH assay, and Smad7/p21/p-Smad2/p-Smad3/E-cad/vimentin expression was assessed by IHC in paraffin-embedded sections of PDX1 tissue samples. $\mathbf{f} \mathrm{A}$ schematic drawing indicates the mechanism by which cESRP1 upregulates Smad7/p21(CDKN1A) expression to inactivate the TGF- $\beta / \mathrm{Smad}$ pathway and inhibit EMT to enhance the chemosensitivity of SCLC 
CESRP1 expression is downregulated in SCLC tissues and predicts a poor prognosis

We further analysed whether decreased cESRP1 expression correlates with SCLC patient prognosis. As shown in Fig. 6a, cESRP1 expression was significantly decreased in 106 SCLC patient tissues compared with matched nontumour tissue samples. The results showed that a lower level of cESRP1 expression significantly correlated with extensive disease-SCLC (ED-SCLC) and a worse status (Table 1). Furthermore, Kaplan-Meier survival curves showed that the patients with SCLC and lower cESRP1 expression had poorer OS than the patients with SCLC and higher cESRP1 expression (Fig. 6b).

A subsequent univariate analysis showed that the cESRP1 expression level and SCLC disease stage significantly correlated with OS in SCLC patients. Moreover, multivariate analysis showed that low expression of cESRP1 could be a factor for predicting poor survival when cESRP1 expression, age, sex, disease stage, and smoking history were included (Table 2). These data indicated that decreased cESRP1 expression is associated with SCLC progression and that the cESRP1 level can be used as an independent prognostic marker in patients with SCLC.

We also observed the significant positive correlations between cESRP1 and the mRNA expression of Smad7/p21 (CDKN1A) in the tissue samples by qRT-PCR (Fig. S6a). Subsequently, the IHC results revealed decreased Smad7 and p21(CDKN1A) expression in the SCLC tissue

Table 1 Clinical characteristics of 106 patients with SCLC according to the cESRP1 expression level

\begin{tabular}{llll}
\hline Variable & \multicolumn{2}{l}{ cESRP1 } & \multirow{2}{*}{$p$ value } \\
\cline { 2 - 3 } & Low & High & \\
\hline Age, year, $\leq 62:>62$ & $24: 30$ & $30: 22$ & 0.173 \\
Sex, male:female & $44: 10$ & $42: 9$ & 0.908 \\
Smocking history, Yes:No & $29: 23$ & $24: 22$ & 0.722 \\
Disease stage, LD:ED & $15: 37$ & $29: 17$ & $0.001^{*}$ \\
Status, Survival:Death & $30: 24$ & $40: 12$ & $0.020^{*}$
\end{tabular}

$\chi^{2}$ test was used to test the association between two categorical variables (*represents statistically significant differences $(p<0.05)$ )

$L D$ limited-stage diseases, $E D$ extensive-stage disease samples and were positively correlated with the cESRP1 levels (Fig. 6c, d).

Furthermore, we found that cESRP1 expression was significantly higher in the chemo-naive tissue samples than that in the paired PDX chemoresistant tissue samples, whereas miR-93-5p abundance was similar between the two sets of samples using RNA FISH assay. We also confirmed that the levels of Smad7/p21 (CDKN1A) and the epithelial marker E-cadherin were downregulated in the PDXresistant tissue samples, whereas those of TGF- $\beta$-EMT activated markers including $\mathrm{p}-\mathrm{Smad} 2 / 3$ and vimentin were upregulated in the chemoresistant tissue samples (Fig. 6e; Fig. S6b). Based on these results, we conclude that cESRP1 may regulate Smad7/p21 (CDKN1A) to negatively impact the TGF- $\beta$-EMT signalling pathway in SCLC in vivo.

\section{Discussion}

Increasing evidence suggests that circRNAs are involved in a wide range of biological processes, including the development of cancers [63]. A recent study by Li et al. identified an FLI1 exonic circRNAs that has a functional role in the progression of SCLC [64]. However, chemoresistanceassociated circRNAs in SCLC have rarely been reported. In our present study, we screened several differentially expressed circRNAs in chemotherapy-resistant cells using circRNA microarray and qRT-PCR analyses. Among these circRNAs, we identified a novel SCLC associated circRNA (cESRP1) that is produced from exons 7, 8, and 9 of the ESRP1 gene locus and is significantly downregulated in drug-resistant cells compared with drug-sensitive cells. We then investigated the function of cESRP1 in SCLC chemoresistance and observed that it can improve chemosensitivity in H69/H69AR and H446/H446DDP cells in vitro and in vivo. Importantly, cESRP1 expression was significantly decreased in SCLC tissues compared with that observed in matched non-tumour tissues. Lower cESRP1 expression was positively correlated with the SCLC extensive stages of patients and poorer survival. Our results suggest, for the first time, that cESRP1 may play a role in SCLC chemoresistance and serve as a valuable prognostic biomarker for patients with SCLC.
Table 2 Univariate and multivariate Cox-regression analysis of various prognostic parameters in patients with SCLC (*represents statistically significant differences $(p<0.05))$

\begin{tabular}{|c|c|c|c|c|c|}
\hline \multirow[t]{2}{*}{ Variable } & \multicolumn{2}{|c|}{ Univariate analysis } & \multicolumn{3}{|c|}{ Multivariate analysis } \\
\hline & $p$ & HR & $p$ & HR $(95 \%$ CI $)$ & Coefficient \\
\hline cESRP1 & $0.010^{*}$ & 0.590 & $0.046^{*}$ & $0.66(0.44-0.99)$ & -0.409 \\
\hline Age & 0.724 & 1.007 & 0.914 & $1.00(0.96-1.92)$ & 0.002 \\
\hline Sex & 0.868 & 1.072 & 0.546 & $0.75(0.29-1.92)$ & -0.300 \\
\hline Disease stage & $<0.001 *$ & 4.583 & $<0.001 *$ & $4.33(1.91-9.78)$ & 1.464 \\
\hline Smoking history & 0.478 & 1.276 & 0.6 & $1.22(0.58-2.57)$ & 0.200 \\
\hline
\end{tabular}


Some circRNAs have been well characterised to function as miRNA sponges in tumorigenesis [11, 47, 49, 65-67]. To better understand the potential role of cESRP1 in SCLC chemoresistance, we confirmed that cESRP1 was primarily located in the cytoplasm, which indicated that cESRP1 may function as an miRNA sponge in SCLC. In addition, our previous studies indicated that specific miRNAs are involved in chemoresistance in SCLC [68, 69]. These results prompted us to further explore the interaction between cESRP1 and its associated miRNA in SCLC. We first investigated the presence of cESRP1 seed binding sites for miR-93-5p, miR-182-5p, and miR-125a-5p by combining our previous miRNA microarray results. However, only miR-93-5p was confirmed to mediate multidrug resistance in SCLC. The results of subsequent luciferase assays, a biotinylated miRNA pulldown assay, and rescue experiments further demonstrated that cESRP1 can inhibit chemoresistance in SCLC by adsorbing miR-93-5p. More strikingly, the combined use of an miR-93-5p antagomir and chemotherapeutic drugs could reverse the resistance of tumours to $\mathrm{C} / \mathrm{E}$ chemotherapy.

In this study, we further showed that cESRP1 may function as a ceRNA to regulate $\operatorname{Smad} 7 / \mathrm{p} 21$ (CDKN1A) by sponging miR-93-5p to inhibit SCLC chemoresistance. We also demonstrated that cESRP1 prohibits TGF- $\beta$-mediated EMT via an miR-93-5p-Smad7/p21(CDKN1A) axis. Smad7/p21(CDKN1A) have been shown to act as components in the negative feedback regulation of TGF- $\beta$ signalling [21, 31, 32, 58]. Our results suggested that cESRP1 effectively increases the abundance of Smad7/p21 $(\mathrm{CDKN} 1 \mathrm{~A})$ and formed a double-negative feedback loop to abolish the effect of the TGF- $\beta /$ Smad signalling pathway. A growing number of clinical studies have shown that antiTGF- $\beta$ therapy has an acceptable safety/tolerability profile and exhibits anti-tumour activity in subsets of patients [70-72]. In this study, we used an SCLC chemoresistant cell line orthotopic xenograft model and observed that the TGF- $\beta$ signalling inhibitor LY2157299 (galunisertib), which was bioavailable, effectively suppressed the TGF- $\beta$ signalling pathway, augmenting tumour chemotherapy responsiveness in combination with chemotherapeutic drugs.

It is particularly noteworthy that in our study, we established a progressive chemoresistant PDX model that mimics recurrent chemoresistance during the clinical course of SCLC. We further demonstrated that ectopic cESRP1 overexpression significantly improved the OS of mice in the PDX model. Most importantly, LY2157299 effectively strengthened the inhibition of tumour growth by first-line platinum-based chemotherapy in the chemoresistant PDX model.

In summary, in this study, we provided comprehensive evidence that cESRP1 acts as a suppressor of chemoresistance and is a prognostic biomarker for patients with SCLC.
cESRP1 enhances drug sensitivity via the miR-93-5pSmad7/p21(CDKN1A) axis by inhibiting TGF- $\beta$-mediated EMT (Fig. 6f). We also demonstrated that inhibition of TGF- $\beta$ signalling can diminish tumour growth by a combined treatment with the TGF- $\beta$ signalling inhibitor LY2157299 and chemotherapeutic drugs in SCLC PDX. These findings have significant implications regarding our understanding of the pathogenesis of SCLC multidrug resistance and highlights the importance of investigating the complicated circRNA-miRNA regulatory gene network as well as TGF- $\beta$ signalling in SCLC progression and treatment efficacy.

Acknowledgements This work was supported by the Chinese National Natural Science Fund (No. 81572244, 81772458) and the Clinical Research Initiative Project of Southern Medical University (LC2016ZD029).

\section{Compliance with ethical standards}

Conflict of interest The authors declare that they have no conflict of interest.

Publisher's note Springer Nature remains neutral with regard to jurisdictional claims in published maps and institutional affiliations.

Open Access This article is licensed under a Creative Commons Attribution 4.0 International License, which permits use, sharing, adaptation, distribution and reproduction in any medium or format, as long as you give appropriate credit to the original author(s) and the source, provide a link to the Creative Commons license, and indicate if changes were made. The images or other third party material in this article are included in the article's Creative Commons license, unless indicated otherwise in a credit line to the material. If material is not included in the article's Creative Commons license and your intended use is not permitted by statutory regulation or exceeds the permitted use, you will need to obtain permission directly from the copyright holder. To view a copy of this license, visit http://creativecommons. org/licenses/by/4.0/.

\section{References}

1. Gazdar AF, Bunn PA, Minna JD. Small-cell lung cancer: what we know, what we need to know and the path forward. Nat Rev Cancer. 2017;17:725-37.

2. Bray F, Ferlay J, Soerjomataram I, Siegel RL, Torre LA, Jemal A. Global cancer statistics 2018: GLOBOCAN estimates of incidence and mortality worldwide for 36 cancers in 185 countries. CA Cancer J Clin. 2018;68:394-424.

3. Kim YH, Mishima M. Second-line chemotherapy for small-cell lung cancer (SCLC). Cancer Treat Rev. 2011;37:143-50.

4. Chen L-L. The biogenesis and emerging roles of circular RNAs. Nat Rev Mol Cell Biol. 2016;17:205-11.

5. Cherubini A, Barilani M, Rossi RL, Jalal MMK, Rusconi F, Buono G, et al. FOXP1 circular RNA sustains mesenchymal stem cell identity via microRNA inhibition. Nucleic Acids Res. 2019;47:5325-40.

6. Li X, Zheng Y, Zheng Y, Huang Y, Zhang Y, Jia L, et al. Circular RNA CDR1as regulates osteoblastic differentiation of periodontal ligament stem cells via the miR-7/GDF5/SMAD and p38 MAPK signaling pathway. Stem Cell Res Ther. 2018;9:232. 
7. Szabo L, Morey R, Palpant NJ, Wang PL, Afari N, Jiang C, et al. Statistically based splicing detection reveals neural enrichment and tissue-specific induction of circular RNA during human fetal development. Genome Biol. 2015;16:126.

8. Vo JN, Cieslik M, Zhang Y, Shukla S, Xiao L, Zhang Y, et al. The landscape of circular RNA in cancer. Cell. 2019;176:869-81.e13.

9. Zhong Y, Du Y, Yang X, Mo Y, Fan C, Xiong F, et al. Circular RNAs function as ceRNAs to regulate and control human cancer progression. Mol Cancer. 2018;17:79.

10. Panda AC, Grammatikakis I, Kim KM, De S, Martindale JL, Munk R, et al. Identification of senescence-associated circular RNAs (SAC-RNAs) reveals senescence suppressor CircPVT1. Nucleic Acids Res. 2017;45:4021-35.

11. Cheng X, Zhang L, Zhang K, Zhang G, Hu Y, Sun X, et al. Circular RNA VMA21 protects against intervertebral disc degeneration through targeting miR-200c and X linked inhibitorof-apoptosis protein. Ann Rheum Dis. 2018;77:770-9.

12. Qian L, Yu S, Chen Z, Meng Z, Huang S, Wang P. The emerging role of circRNAs and their clinical significance in human cancers. Biochim Biophys Acta Rev Cancer. 2018;1870:247-60.

13. Yang W, Gu J, Wang X, Wang Y, Feng M, Zhou D, et al. Inhibition of circular RNA CDR1as increases chemosensitivity of 5FU-resistant BC cells through up-regulating miR-7. J Cell Mol Med. 2019;23:3166-77.

14. Huang X, Li Z, Zhang Q, Wang W, Li B, Wang L, et al. Circular RNA AKT3 upregulates PIK3R1 to enhance cisplatin resistance in gastric cancer via miR-198 suppression. Mol Cancer. 2019;18:71.

15. Seoane J, Gomis RR. TGF-beta family signaling in tumor suppression and cancer progression. Cold Spring Harb Perspect Biol. 2017;9:a022277.

16. Cao J, Song Y, Bi N, Shen J, Liu W, Fan J, et al. DNA methylation-mediated repression of miR-886-3p predicts poor outcome of human small cell lung cancer. Cancer Res. 2013;73:3326-35.

17. Derynck R, Zhang YE. Smad-dependent and Smad-independent pathways in TGF-beta family signalling. Nature. 2003;425: 577-84.

18. Kit Leng Lui S, Iyengar PV, Jaynes P, Isa ZFBA, Pang B, Tan TZ, et al. USP2 6 regulates TGF- $\beta$ signaling by deubiquitinating and stabilizing SMAD7. EMBO Rep. 2017;18:797-808.

19. Miyazono K. Positive and negative regulation of TGF-beta signaling. J Cell Sci. 2000;113(Pt 7):1101-9.

20. Yan X, Liao H, Cheng M, Shi X, Lin X, Feng X, et al. Smad7 protein interacts with receptor-regulated Smads (R-Smads) to inhibit transforming growth factor- $\beta$ (TGF- $\beta$ )/Smad signaling. J Biol Chem. 2016;291:382-92.

21. Zhang S, Fei T, Zhang L, Zhang R, Chen F, Ning Y, et al. Smad7 antagonizes transforming growth factor beta signaling in the nucleus by interfering with functional Smad-DNA complex formation. Mol Cell Biol. 2007;27:4488-99.

22. Seoane J. p21(WAF1/Cip1) at the switch between the anti oncogenic and oncogenic faces of TGF beta. Cancer Biol Ther. 2004;3:226-7.

23. Zhang Q, Xiao M, Gu S, Xu Y, Liu T, Li H, et al. ALK phosphorylates SMAD4 on tyrosine to disable TGF- $\beta$ tumour suppressor functions. Nat Cell Biol. 2019;21:179-89.

24. Yeh HW, Hsu EC, Lee SS, Lang YD, Lin YC, Chang CY, et al. PSPC1 mediates TGF-beta1 autocrine signalling and Smad2/3 target switching to promote EMT, stemness and metastasis. Nat Cell Biol. 2018;20:479-91.

25. Cai Z, Cao Y, Luo Y, Hu H, Ling H. Signalling mechanism(s) of epithelial-mesenchymal transition and cancer stem cells in tumour therapeutic resistance. Clin Chim Acta. 2018;483:156-63.

26. Fischer KR, Durrans A, Lee S, Sheng J, Li F, Wong ST, et al. Epithelial-to-mesenchymal transition is not required for lung metastasis but contributes to chemoresistance. Nature. 2015;527:472-6.
27. Brown JA, Yonekubo Y, Hanson N, Sastre-Perona A, Basin A, Rytlewski JA, et al. TGF-beta-Induced quiescence mediates chemoresistance of tumor-propagating cells in squamous cell carcinoma. Cell Stem Cell. 2017;21:650-64 e8.

28. Song B, Park SH, Zhao JC, Fong KW, Li S, Lee Y, et al. Targeting FOXA1-mediated repression of TGF-beta signaling suppresses castration-resistant prostate cancer progression. J Clin Invest. 2019;129:569-82.

29. Li H, Li J, Chen L, Qi S, Yu S, Weng Z, et al. HERC3-mediated SMAD7 ubiquitination degradation promotes autophagy-induced EMT and chemoresistance in glioblastoma. Clin Cancer Res. 2019;25:3602-16.

30. Huang S, Holzel M, Knijnenburg T, Schlicker A, Roepman P, McDermott $U$, et al. MED12 controls the response to multiple cancer drugs through regulation of TGF-beta receptor signaling. Cell. 2012;151:937-50.

31. Bauer J, Ozden O, Akagi N, Carroll T, Principe DR, Staudacher $\mathrm{JJ}$, et al. Activin and TGF $\beta$ use diverging mitogenic signaling in advanced colon cancer. Mol Cancer. 2015;14:182.

32. Liu M, Casimiro MC, Wang C, Shirley LA, Jiao X, Katiyar S, et al. p21CIP1 attenuates Ras- and c-Myc-dependent breast tumor epithelial mesenchymal transition and cancer stem cell-like gene expression in vivo. Proc Natl Acad Sci USA. 2009;106:19035-9.

33. Mutlu M, Raza U, Saatci Ö, Eyüpoğlu E, Yurdusev E, Şahin Ö. miR-200c: a versatile watchdog in cancer progression, EMT, and drug resistance. J Mol Med (Berl). 2016;94:629-44.

34. Gulei D, Mehterov N, Ling H, Stanta G, Braicu C, BerindanNeagoe I. The "good-cop bad-cop" TGF-beta role in breast cancer modulated by non-coding RNAs. Biochim Biophys Acta Gen Subj. 2017;1861:1661-75.

35. Li LQ, Pan D, Chen Q, Zhang SW, Xie DY, Zheng XL, et al. Sensitization of gastric cancer cells to 5-FU by microRNA-204 through targeting the TGFBR2-mediated epithelial to mesenchymal transition. Cell Physiol Biochem. 2018;47:1533-45.

36. Wang L, Tong X, Zhou Z, Wang S, Lei Z, Zhang T, et al. Circular RNA hsa_circ_0008305 (circPTK2) inhibits TGF- $\beta$-induced epithelial-mesenchymal transition and metastasis by controlling TIF1 $\gamma$ in non-small cell lung cancer. Mol Cancer. 2018;17:140.

37. Tan SM, Lieberman J. Capture and identification of miRNA targets by biotin pulldown and RNA-seq. Methods Mol Biol. 2016;1358:211-28.

38. Huang W, Su G, Huang X, Zou A, Wu J, Yang Y, et al. Long noncoding RNA PCAT6 inhibits colon cancer cell apoptosis by regulating anti-apoptotic protein ARC expression via EZH2. Cell Cycle. 2019;18:69-83.

39. Gao X, Gulari E, Zhou X. In situ synthesis of oligonucleotide microarrays. Biopolymers. 2004;73:579-96.

40. Anderson WC, Boyd MB, Aguilar J, Pickell B, Laysang A, Pysz $\mathrm{MA}$, et al. Initiation and characterization of small cell lung cancer patient-derived xenografts from ultrasound-guided transbronchial needle aspirates. PLoS ONE. 2015;10:e0125255.

41. Drapkin BJ, George J, Christensen CL, Mino-Kenudson M, Dries $\mathrm{R}$, Sundaresan T, et al. Genomic and functional fidelity of small cell lung cancer patient-derived xenografts. Cancer Disco. 2018;8:600-15.

42. Gardner EE, Lok BH, Schneeberger VE, Desmeules P, Miles LA, Arnold PK, et al. Chemosensitive relapse in small cell lung cancer proceeds through an EZH2-SLFN11 axis. Cancer Cell. 2017;31: 286-99.

43. Dalerba P, Dylla SJ, Park IK, Liu R, Wang X, Cho RW, et al. Phenotypic characterization of human colorectal cancer stem cells. Proc Natl Acad Sci USA. 2007;104:10158-63.

44. Al-Hajj M, Wicha MS, Benito-Hernandez A, Morrison SJ, Clarke MF. Prospective identification of tumorigenic breast cancer cells. Proc Natl Acad Sci USA. 2003;100:3983-8. 
45. Jeong HM, Han J, Lee SH, Park HJ, Lee HJ, Choi JS, et al. ESRP1 is overexpressed in ovarian cancer and promotes switching from mesenchymal to epithelial phenotype in ovarian cancer cells. Oncogenesis. 2017;6:e389.

46. Walser TC, Jing Z, Tran LM, Lin YQ, Yakobian N, Wang G, et al. Silencing the snail-dependent RNA splice regulator ESRP1 drives malignant transformation of human pulmonary epithelial cells. Cancer Res. 2018;78:1986-99.

47. Yu J, Xu QG, Wang ZG, Yang Y, Zhang L, Ma JZ, et al. Circular RNA cSMARCA5 inhibits growth and metastasis in hepatocellular carcinoma. J Hepatol. 2018;68:1214-27.

48. Guo L, Liu Y, Bai Y, Sun Y, Xiao F, Guo Y. Gene expression profiling of drug-resistant small cell lung cancer cells by combining microRNA and cDNA expression analysis. Eur J Cancer. 2010;46:1692-702.

49. Wang L, Liang Y, Mao Q, Xia W, Chen B, Shen H, et al. Circular RNA circCRIM1 inhibits invasion and metastasis in lung adenocarcinoma through the microRNA (miR)-182/miR-93-leukemia inhibitory factor receptor pathway. Cancer Sci. 2019; 110:2960-72.

50. Xiao X, Zhou L, Cao P, Gong H, Zhang Y. MicroRNA-93 regulates cyclin $\mathrm{G} 2$ expression and plays an oncogenic role in laryngeal squamous cell carcinoma. Int J Oncol. 2015;46:161-74.

51. Chen J, Deng Y, Ao L, Song Y, Xu Y, Wang CC, et al. The highrisk HPV oncogene E7 upregulates miR-182 expression through the TGF- $\beta /$ Smad pathway in cervical cancer. Cancer Lett. 2019;460:75-85.

52. Li X, Xu Y, Ding Y, Li C, Zhao H, Wang J, et al. Posttranscriptional upregulation of HER3 by HER2 mRNA induces trastuzumab resistance in breast cancer. Mol Cancer. 2018;17:113.

53. Petrocca F, Visone R, Onelli MR, Shah MH, Nicoloso MS, de Martino I, et al. E2F1-regulated microRNAs impair TGFbetadependent cell-cycle arrest and apoptosis in gastric cancer. Cancer Cell. 2008;13:272-86.

54. Smith AL, Iwanaga R, Drasin DJ, Micalizzi DS, Vartuli RL, Tan AC, et al. The miR-106b-25 cluster targets Smad7, activates TGF$\beta$ signaling, and induces EMT and tumor initiating cell characteristics downstream of Six 1 in human breast cancer. Oncogene. 2012;31:5162-71.

55. Oshimori N, Oristian D, Fuchs E. TGF- $\beta$ promotes heterogeneity and drug resistance in squamous cell carcinoma. Cell. 2015; 160:963-76.

56. Yu Y, Feng XH. TGF- $\beta$ signaling in cell fate control and cancer. Curr Opin Cell Biol. 2019;61:56-63.

57. Hao Y, Baker D, Ten Dijke P. TGF- $\beta$-mediated epithelialmesenchymal transition and cancer metastasis. Int $\mathrm{J}$ Mol Sci. 2019;20:2767-801.

58. Hayashi H, Abdollah S, Qiu Y, Cai J, Xu YY, Grinnell BW, et al. The MAD-related protein Smad7 associates with the TGFbeta receptor and functions as an antagonist of TGFbeta signaling. Cell. 1997;89:1165-73.
59. Yu J, Lei R, Zhuang X, Li X, Li G, Lev S, et al. MicroRNA-182 targets SMAD7 to potentiate TGF $\beta$-induced epithelial-mesenchymal transition and metastasis of cancer cells. Nat Commun. 2016;7:13884.

60. Seoane J, Le HV, Shen L, Anderson SA, Massagué J. Integration of Smad and forkhead pathways in the control of neuroepithelial and glioblastoma cell proliferation. Cell. 2004;117:211-23.

61. Koinuma D, Tsutsumi S, Kamimura N, Taniguchi H, Miyazawa $\mathrm{K}$, Sunamura $\mathrm{M}$, et al. Chromatin immunoprecipitation on microarray analysis of $\operatorname{Smad} 2 / 3$ binding sites reveals roles of ETS1 and TFAP2A in transforming growth factor beta signaling. Mol Cell Biol. 2009;29:172-86.

62. Liu WT, Huang KY, Lu MC, Huang HL, Chen CY, Cheng YL, et al. TGF- $\beta$ upregulates the translation of USP15 via the PI3K/AKT pathway to promote p53 stability. Oncogene. 2017; 36:2715-23.

63. Geng Y, Jiang J, Wu C. Function and clinical significance of circRNAs in solid tumors. J Hematol Oncol. 2018;11:98.

64. Li L, Li W, Chen N, Zhao H, Xu G, Zhao Y, et al. FLI1 exonic circular RNAs as a novel oncogenic driver to promote tumor metastasis in small cell lung cancer. Clin Cancer Res. 2019; 25:1302-17.

65. Cortés-López M, Miura P. Emerging functions of circular RNAs. Yale J Biol Med. 2016;89:527-37.

66. Jeck WR, Sorrentino JA, Wang K, Slevin MK, Burd CE, Liu J, et al. Circular RNAs are abundant, conserved, and associated with ALU repeats. RNA. 2013;19:141-57.

67. Memczak S, Jens M, Elefsinioti A, Torti F, Krueger J, Rybak A, et al. Circular RNAs are a large class of animal RNAs with regulatory potency. Nature. 2013;495:333-8.

68. Fang S, Zeng X, Zhu W, Tang R, Chao Y, Guo L. Zinc finger Ebox-binding homeobox 2 (ZEB2) regulated by miR-200b contributes to multi-drug resistance of small cell lung cancer. Exp Mol Pathol. 2014;96:438-44.

69. Liu H, Wu X, Huang J, Peng J, Guo L. miR-7 modulates chemoresistance of small cell lung cancer by repressing MRP1/ ABCC1. Int J Exp Pathol. 2015;96:240-7.

70. Yingling JM, McMillen WT, Yan L, Huang H, Sawyer JS, Graff $\mathrm{J}$, et al. Preclinical assessment of galunisertib (LY2157299 monohydrate), a first-in-class transforming growth factor- $\beta$ receptor type I inhibitor. Oncotarget. 2018;9:6659-77.

71. Ikeda M, Takahashi H, Kondo S, Lahn MMF, Ogasawara K, Benhadji KA, et al. Phase 1b study of galunisertib in combination with gemcitabine in Japanese patients with metastatic or locally advanced pancreatic cancer. Cancer Chemother Pharm. 2017;79:1169-77.

72. Herbertz S, Sawyer JS, Stauber AJ, Gueorguieva I, Driscoll KE, Estrem ST, et al. Clinical development of galunisertib (LY2157299 monohydrate), a small molecule inhibitor of transforming growth factor-beta signaling pathway. Drug Des Devel Ther. 2015;9:4479-99. 